Revised version

October 2008

\title{
HIGH-ENERGY HADRON-HADRON (DIPOLE-DIPOLE) SCATTERING FROM LATTICE QCD
}

\author{
Matteo Giordand* and Enrico Meggiolard \\ Dipartimento di Fisica, Università di Pisa, and INFN, Sezione di Pisa, \\ Largo Pontecorvo 3, I-56127 Pisa, Italy.
}

\begin{abstract}
In this paper the problem of high-energy hadron-hadron (dipole-dipole) scattering is approached (for the first time) from the point of view of lattice QCD, by means of Monte Carlo numerical simulations. In the first part, we give a brief review of how high-energy scattering amplitudes can be reconstructed, using a functional-integral approach, in terms of certain correlation functions of two Wilson loops and we also briefly recall some relevant analyticity and crossing-symmetry properties of these loop-loop correlation functions, when going from Euclidean to Minkowskian theory. In the second part, we shall see how these (Euclidean) loop-loop correlation functions can be evaluated in lattice QCD and we shall compare our numerical results with some nonperturbative analytical estimates that appeared in the literature, discussing in particular the question of the analytic continuation from Euclidean to Minkowskian theory and its relation to the still unsolved problem of the asymptotic $s$-dependence of the hadron-hadron total cross sections.
\end{abstract}

\footnotetext{
*E-mail: matteo.giordano@df.unipi.it

${ }^{\dagger}$ E-mail: enrico.meggiolaro@df.unipi.it
} 


\section{Introduction}

One of the most important open problems in hadronic physics (studied since long before the discovery of QCD) is to explain/predict the (asymptotic) high-energy behaviour of hadron-hadron total cross sections. Present-day experimental observations (up to center-of-mass total energy $\sqrt{s}=1.8 \mathrm{TeV}$ ) seem to be well described by a pomeron-like high-energy behaviour (see, for example, Ref. [1] and references therein):

$$
\sigma_{\text {tot }}^{(h h)}(s) \underset{s \rightarrow \infty}{\sim} \sigma_{0}^{(h h)}\left(\frac{s}{s_{0}}\right)^{\epsilon_{P}}, \quad \text { with } \epsilon_{P} \simeq 0.08
$$

This behaviour is known in the literature as the soft pomeron, to be distinguished from the well-known BFKL (or hard) pomeron [2], i.e., $\sim s^{\frac{12 \alpha_{s}}{\pi} \log 2}$, with $\alpha_{s}=g^{2} / 4 \pi$, obtained in perturbative QCD. As we believe QCD to be the fundamental theory of strong interactions, we also expect that it correctly predicts from first principles the behaviour of hadronic total cross sections with energy. Anyway, in spite of all the efforts, a satisfactory solution to this problem is still lacking.

We should also remind the reader at this point that the pomeron-like behaviour (1.1) is, strictly speaking, theoretically forbidden (at least if considered as a true asymptotic behaviour) by the well-known Froissart-Lukaszuk-Martin (FLM) theorem [3] (see also [4]),

according to which, for $s \rightarrow \infty, \sigma_{\text {tot }}(s) \leq \frac{\pi}{m_{\pi}^{2}} \log ^{2}\left(\frac{s}{s_{0}}\right)$, where $m_{\pi}$ is the pion mass and $s_{0}$ is an unspecified squared mass scale. In this respect, the pomeron-like behaviour (1.1) can at most be regarded as a sort of pre-asymptotic (but not really asymptotic!) behaviour of the high-energy total cross sections (see, e.g., Refs. [5, 6, 7] and references therein), valid in a certain high-energy range.

From a general theoretical point of view, the optical theorem (which is a consequence of unitarity) allows one to derive hadron-hadron total cross sections from the expressions of the hadron-hadron elastic scattering amplitudes $\mathcal{M}_{(h h)}(s, t)$ ( $t$ being the transferred momentum squared):

$$
\sigma_{\text {tot }}^{(h h)}(s) \underset{s \rightarrow \infty}{\sim} \frac{1}{s} \operatorname{Im} \mathcal{M}_{(h h)}(s, t=0) .
$$

High-energy hadron-hadron elastic scattering processes at low transferred momentum (usually called soft high-energy scattering processes) possess two different and widely separated energy scales: the center-of-mass total energy squared $s$, which is a hard scale $\left(s \gg 1 \mathrm{GeV}^{2}\right.$ : formally we consider the limit $\left.s \rightarrow \infty\right)$, and the transferred momentum 
squared $t$, which is a soft scale, smaller than (or nearly equal to) the typical energy scale of strong interactions $\left(|t| \lesssim 1 \mathrm{GeV}^{2} \ll s\right)$. In this energy regime we cannot fully rely on perturbation theory and a genuine nonperturbative treatment is in order.

Since Nachtmann's seminal paper in 1991 [8] a lot of work has been done on the problem of soft high-energy scattering in the framework of nonperturbative QCD [9, 10, 11, 12, 13. Using a functional-integral approach, hadron-hadron elastic scattering amplitudes are reconstructed from the correlation functions of certain Wilson loops, defined in Minkowski space-time (see Section 2 below). As it has been shown in [14, 15, 16, 17] (see Section 2 below), such correlation functions can be recovered after proper analytic continuation from their Euclidean counterparts, i.e., correlation functions of certain Wilson loops forming an angle $\theta$ in Euclidean space: this has paved the way to the application of nonperturbative techniques, which are normally available only in the Euclidean formulation of QCD. Some calculations of the loop-loop Euclidean correlation functions already exist in the literature (see Section 4 below), using the so-called Stochastic Vacuum Model (SVM) [18, the Instanton Liquid Model (ILM) [19], or the AdS/CFT correspondence for strongly coupled gauge theories in the limit of a large number of colours [20, 21, 22]. The Euclidean correlator is then continued to the corresponding Minkowskian correlation function using the above-mentioned analytic continuation in the angular variables. The loop-loop correlation functions (both in the Minkowskian and in the Euclidean theories) have also been computed exactly in the first two orders of perturbation theory, $\mathcal{O}\left(g^{4}\right)$ and $\mathcal{O}\left(g^{6}\right)$, in Ref. [5].

Of course, each of these models, used in the calculation of the Euclidean correlation functions, has its own limitations, which are reflected in the variety of answers in the literature: someone finds constant cross sections, someone else finds a soft-pomeron behaviour, someone else finds a hard-pomeron behaviour ... (And maybe the true asymptotic behaviour is $\log ^{2}\left(s / s_{0}\right)$, thus saturating the FLM bound!?). Unfortunately, these limitations are often out of control, in the sense that one does not know how much information is lost due to the involved approximations. This is surely a crucial point which, in our opinion, should be further investigated. (For example, in Ref. [23] it has been shown that the Euclidean-to-Minkowskian analytic-continuation approach can, with the inclusion of some extra, more or less plausible, assumptions, easily reproduce a pre-asymptotic pomeron-like behaviour, like the one in (1.1), which violates the FLM bound.)

A real breakthrough in this direction could be provided by a direct numerical calculation of the loop-loop Euclidean correlation function in Lattice Gauge Theory (LGT). 
In particular, one is interested in the dependence of the correlation function on the angle $\theta$ between the loops, from which the energy dependence of the physical scattering amplitudes can be derived after a proper analytic continuation to Minkowski space-time. Clearly a lattice approach can at most give the above-mentioned function only for a finite set of $\theta$-values, from which it is clearly impossible (without some extra assumption on the interpolating continuous function) to get, by analytic continuation, the corresponding Minkowskian correlation function (and, from this, the elastic scattering amplitudes and the total cross sections). However, the lattice approach, which provides a real firstprinciple determination of the loop-loop correlator in Euclidean space, can be used to investigate the goodness of a given existing analytic model (such as SVM, ILM, AdS/CFT, and so on ...) or even to open the way to some new model, simply by trying to fit the lattice data with the considered model. This is exactly what we shall try to do in this paper.

The plan of this paper is the following. In Section 2 we give (for the benefit of the reader) a brief review of how high-energy scattering amplitudes can be reconstructed, using a functional-integral approach, in terms of certain correlation functions of two Wilson loops and we also briefly recall some relevant analyticity and crossing-symmetry properties of these loop-loop correlation functions, when going from Euclidean to Minkowskian theory. In Section 3 we shall see how these (Euclidean) loop-loop correlation functions can be evaluated in lattice QCD and discuss the main technical complications. In Section 4 we shall compare our numerical results with some analytical nonperturbative estimates that appeared in the literature, discussing in particular the question of the analytic continuation from Euclidean to Minkowskian theory and its relation to the still unsolved problem of the asymptotic $s$-dependence of the hadron-hadron total cross sections. In Section 5 we draw our conclusions and show some prospects for the future.

\section{Hadron-hadron (dipole-dipole) scattering amplitudes from Wilson loop correlators}

It was shown in Refs. [9, 10, 11] (for a review see Refs. [1, 12]) that the high-energy

meson-meson elastic scattering amplitude can be approximately reconstructed in two steps. 
i) One first evaluates, in the functional-integral approach, the high-energy elastic scattering amplitude $\mathcal{M}_{(d d)}$ of two $q \bar{q}$ pairs (usually called dipoles) of given transverse sizes $\vec{R}_{1 \perp}$ and $\vec{R}_{2 \perp}$ and given longitudinal-momentum fractions $f_{1}$ and $f_{2}$ of the two quarks in the two dipoles respectively. This dipole-dipole scattering amplitude turns out to be governed by the (properly normalised) correlation function of two Wilson loops $\mathcal{W}_{1}$ and $\mathcal{W}_{2}$, which follow the classical straight lines for quark (antiquark) trajectories:

$$
\mathcal{M}_{(d d)}\left(s, t ; \vec{R}_{1 \perp}, f_{1}, \vec{R}_{2 \perp}, f_{2}\right) \equiv-i 2 s \int d^{2} \vec{z}_{\perp} e^{i \vec{q}_{\perp} \cdot \vec{z}_{\perp}}\left[\frac{\left\langle\mathcal{W}_{1} \mathcal{W}_{2}\right\rangle}{\left\langle\mathcal{W}_{1}\right\rangle\left\langle\mathcal{W}_{2}\right\rangle}-1\right]
$$

where $s$ and $t=-\left|\vec{q}_{\perp}\right|^{2}\left(\vec{q}_{\perp}\right.$ being the transferred momentum) are the usual Mandelstam variables. The expectation values $\left\langle\mathcal{W}_{1} \mathcal{W}_{2}\right\rangle,\left\langle\mathcal{W}_{1}\right\rangle,\left\langle\mathcal{W}_{2}\right\rangle$ are averages in the sense of the QCD functional integral $\left(\langle\mathcal{O}[A]\rangle \equiv \frac{1}{Z} \int[d A] \mathcal{O}[A] \operatorname{det} \mathcal{Q}[A] e^{i S[A]}, Z \equiv \int[d A] \operatorname{det} \mathcal{Q}[A] e^{i S[A]}\right.$, with $S[A]$ the pure-gauge action and $\operatorname{det} \mathcal{Q}[A]$ the fermion-matrix determinant) and the Wilson loops $\mathcal{W}_{1}$ and $\mathcal{W}_{2}$ are defined as follows (for $N_{c}$-colour QCD):

$$
\mathcal{W}_{1,2}^{(T)} \equiv \frac{1}{N_{c}} \operatorname{Tr}\left\{\mathcal{P} \exp \left[-i g \oint_{\mathcal{C}_{1,2}} A_{\mu}(x) d x^{\mu}\right]\right\}
$$

where $\mathcal{P}$ denotes the path ordering along the given path $\mathcal{C}$ and $A_{\mu}=A_{\mu}^{a} T^{a}, T^{a}$ being the generators of the $S U\left(N_{c}\right)$ Lie algebra in the fundamental representation; $\mathcal{C}_{1}$ and $\mathcal{C}_{2}$ are two rectangular paths which follow the classical straight lines for the quark $\left[X_{q}(\tau)\right.$, forward in proper time $\tau]$ and the antiquark $\left[X_{\bar{q}}(\tau)\right.$, backward in $\left.\tau\right]$ trajectories, i.e.,

$$
\begin{aligned}
& \mathcal{C}_{1}: \quad X_{1 q}^{\mu}(\tau)=z^{\mu}+\frac{p_{1}^{\mu}}{m} \tau+\left(1-f_{1}\right) R_{1}^{\mu}, \quad X_{1 \bar{q}}^{\mu}(\tau)=z^{\mu}+\frac{p_{1}^{\mu}}{m} \tau-f_{1} R_{1}^{\mu}, \\
& \mathcal{C}_{2}: \quad X_{2 q}^{\mu}(\tau)=\frac{p_{2}^{\mu}}{m} \tau+\left(1-f_{2}\right) R_{2}^{\mu}, \quad X_{2 \bar{q}}^{\mu}(\tau)=\frac{p_{2}^{\mu}}{m} \tau-f_{2} R_{2}^{\mu},
\end{aligned}
$$

and are closed by straight-line paths at proper times $\tau= \pm T$, where $T$ plays the role of an infrared (IR) cutoff [24, 16], which can and must be removed in the end, by letting $T \rightarrow \infty$. (In fact, differently from the parton-parton scattering amplitudes, which are known to be affected by IR divergences, the elastic scattering amplitude of two colourless states in gauge theories, e.g., two $q \bar{q}$ meson states, is expected to be an IR-finite physical quantity [25].)

Here $p_{1}$ and $p_{2}$ are the four-momenta of the two dipoles, taken for simplicity with the 
same mass $m$, moving (in the center-of-mass frame) with speed $V$ and $-V$ along, for example, the $x^{1}$-direction:

$$
p_{1}=m\left(\cosh \frac{\chi}{2}, \sinh \frac{\chi}{2}, \overrightarrow{0}_{\perp}\right), \quad p_{2}=m\left(\cosh \frac{\chi}{2},-\sinh \frac{\chi}{2}, \overrightarrow{0}_{\perp}\right)
$$

Here $\chi=2 \operatorname{arctanh} V$ is the hyperbolic angle between the two trajectories $1 q$ and $2 q$, i.e., $p_{1} \cdot p_{2}=m^{2} \cosh \chi$. In the high-energy limit $s \rightarrow \infty$ (i.e., $\chi \rightarrow+\infty$ ),

$$
s \equiv\left(p_{1}+p_{2}\right)^{2}=2 m^{2}(\cosh \chi+1), \quad \text { i.e. : } \chi \underset{s \rightarrow \infty}{\sim} \log \left(\frac{s}{m^{2}}\right)
$$

Moreover, $R_{1}=\left(0,0, \vec{R}_{1 \perp}\right), R_{2}=\left(0,0, \vec{R}_{2 \perp}\right)$ and $z=\left(0,0, \vec{z}_{\perp}\right)$, where $\vec{z}_{\perp}=\left(z^{2}, z^{3}\right)$ is the impact-parameter distance between the two loops in the transverse plane. The two Wilson loops are schematically shown in Fig. 1.

If we introduce the following notation for the normalised connected loop-loop correlator (in the presence of a finite IR cutoff $T$ ), *

$$
\mathcal{G}_{M}\left(\chi ; T ; \vec{z}_{\perp} ; 1,2\right) \equiv \frac{\left\langle\mathcal{W}_{1}^{(T)} \mathcal{W}_{2}^{(T)}\right\rangle}{\left\langle\mathcal{W}_{1}^{(T)}\right\rangle\left\langle\mathcal{W}_{2}^{(T)}\right\rangle}-1
$$

where the arguments " 1 " and " 2 " stand for " $\vec{R}_{1 \perp}, f_{1}$ " and " $\vec{R}_{2 \perp}, f_{2}$ " respectively, the dipole-dipole scattering amplitude (2.1) can be rewritten as

$$
\begin{aligned}
& \mathcal{M}_{(d d)}\left(s, t ; \vec{R}_{1 \perp}, f_{1}, \vec{R}_{2 \perp}, f_{2}\right)= \\
& \quad-i 2 s \int d^{2} \vec{z}_{\perp} e^{i \vec{q}_{\perp} \cdot \vec{z}_{\perp}} \mathcal{G}_{M}\left(\chi \underset{s \rightarrow \infty}{\sim} \log \left(\frac{s}{m^{2}}\right) ; T \rightarrow+\infty ; \vec{z}_{\perp} ; 1,2\right) .
\end{aligned}
$$

ii) The hadron-hadron (in our case meson-meson) elastic scattering amplitude $\mathcal{M}_{(h h)}$ can then be obtained by averaging the dipole-dipole scattering amplitude (2.7) over all possible dipole transverse separations $\vec{R}_{1 \perp}$ and $\vec{R}_{2 \perp}$ and longitudinal-momentum fractions $f_{1}$ and $f_{2}$ with two proper squared hadron wave functions $\left|\psi_{1}\left(\vec{R}_{1 \perp}, f_{1}\right)\right|^{2}$ and $\left|\psi_{2}\left(\vec{R}_{2 \perp}, f_{2}\right)\right|^{2}$, describing the two interacting mesons:

$$
\begin{aligned}
\mathcal{M}_{(h h)}(s, t) & =\int d^{2} \vec{R}_{1 \perp} \int_{0}^{1} d f_{1}\left|\psi_{1}\left(\vec{R}_{1 \perp}, f_{1}\right)\right|^{2} \int d^{2} \vec{R}_{2 \perp} \int_{0}^{1} d f_{2}\left|\psi_{2}\left(\vec{R}_{2 \perp}, f_{2}\right)\right|^{2} \\
& \times \mathcal{M}_{(d d)}\left(s, t ; \vec{R}_{1 \perp}, f_{1}, \vec{R}_{2 \perp}, f_{2}\right) .
\end{aligned}
$$

\footnotetext{
*The quantity $\mathcal{G}_{M}$ in Eq. (2.6) is equal to the quantity $\mathcal{G}_{M}-1$ of Refs. [16, 17, 23, 26].
} 
(For the treatment of baryons, a similar, but, of course, more involved, picture can be adopted, using a genuine three-body configuration or, alternatively and even more simply, a quark-diquark configuration: we refer the interested reader to the above-mentioned original references [1, 9, 10, 11, 12.)

All the above refers to the theory in the (physical) Minkowski space-time. However, as we have already observed in the Introduction, most of the nonperturbative methods in field theory are available only in the functional-integral approach formulated in Euclidean space: therefore we are interested in the problem of the analytic continuation of the looploop correlation functions into/from Euclidean space. In the Euclidean theory we can consider the correlation function of two Euclidean Wilson loops $\widetilde{\mathcal{W}}_{1}$ and $\widetilde{\mathcal{W}}_{2}$, defined in the same way as they are in Minkowski space-time, taking into account that the metric is now Euclidean rather than Minkowskian, and running along two rectangular paths $\widetilde{\mathcal{C}}_{1}$ and $\widetilde{\mathcal{C}}_{2}$ which follow the following straight-line trajectories

$$
\begin{aligned}
& \widetilde{\mathcal{C}_{1}}: X_{E \mu}^{1 q}(\tau)=z_{E \mu}+\frac{p_{1 E \mu}}{m} \tau+\left(1-f_{1}\right) R_{1 E \mu}, \quad X_{E \mu}^{1 \bar{q}}(\tau)=z_{E \mu}+\frac{p_{1 E \mu}}{m} \tau-f_{1} R_{1 E \mu}, \\
& \widetilde{\mathcal{C}_{2}}: \quad X_{E \mu}^{2 q}(\tau)=\frac{p_{2 E \mu}}{m} \tau+\left(1-f_{2}\right) R_{2 E \mu}, \quad X_{E \mu}^{2 \bar{q}}(\tau)=\frac{p_{2 E \mu}}{m} \tau-f_{2} R_{2 E \mu},
\end{aligned}
$$

and are closed by straight-line paths at proper times $\tau= \pm T$. Here the Euclidean coordinates are $X_{E \mu}=\left(X_{E 1}, X_{E 2}, X_{E 3}, X_{E 4}\right)$, where $X_{E 4}$ corresponds to the "Euclidean time", and $R_{1 E}=\left(0, \vec{R}_{1 \perp}, 0\right), R_{2 E}=\left(0, \vec{R}_{2 \perp}, 0\right)$ and $z_{E}=\left(0, \vec{z}_{\perp}, 0\right)$, where $\vec{R}_{1 \perp}, \vec{R}_{2 \perp}$ and $\vec{z}_{\perp}$ are exactly the same transverse vectors introduced in the Minkowskian case. Moreover, in the Euclidean theory we choose the four-vectors $p_{1 E}$ and $p_{2 E}$ to be

$$
p_{1 E}=m\left(\sin \frac{\theta}{2}, \overrightarrow{0}_{\perp}, \cos \frac{\theta}{2}\right), \quad p_{2 E}=m\left(-\sin \frac{\theta}{2}, \overrightarrow{0}_{\perp}, \cos \frac{\theta}{2}\right),
$$

$\theta$ being the angle formed by the two trajectories $1 q$ and $2 q$ in Euclidean four-space, i.e., $p_{1 E} \cdot p_{2 E}=m^{2} \cos \theta$.

It turns out [16, 17] that the Minkowskian quantity $\mathcal{G}_{M}\left[\right.$ with $\chi \in \mathbb{R}^{+}$] can be reconstructed from the corresponding Euclidean quantity [with $\theta \in(0, \pi)$ ]

$$
\mathcal{G}_{E}\left(\theta ; T ; \vec{z}_{\perp} ; 1,2\right) \equiv \frac{\left\langle\widetilde{\mathcal{W}}_{1}^{(T)} \widetilde{\mathcal{W}}_{2}^{(T)}\right\rangle}{\left\langle\widetilde{\mathcal{W}}_{1}^{(T)}\right\rangle\left\langle\widetilde{\mathcal{W}}_{2}^{(T)}\right\rangle}-1
$$

(where $\langle\ldots\rangle$ are now averages in the sense of the Euclidean functional integral, i.e., $\left\langle\mathcal{O}\left[A_{E}\right]\right\rangle \equiv \frac{1}{Z_{E}} \int\left[d A_{E}\right] \mathcal{O}\left[A_{E}\right] \operatorname{det} \mathcal{Q}_{E}\left[A_{E}\right] e^{-S_{E}\left[A_{E}\right]}, Z_{E} \equiv \int\left[d A_{E}\right] \operatorname{det} \mathcal{Q}_{E}\left[A_{E}\right] e^{-S_{E}\left[A_{E}\right]}$, with 
$S_{E}\left[A_{E}\right]$ the pure-gauge Euclidean action and $\operatorname{det} \mathcal{Q}_{E}\left[A_{E}\right]$ the Euclidean fermion-matrix determinant $\dagger$ by an analytic continuation in the angular variables $\theta \rightarrow-i \chi$ and in the IR cutoff $T \rightarrow i T$, exactly as in the case of Wilson lines [14, 15, 16]. This result [16, 17] is derived under certain hypotheses of analyticity in the angular variables and in the IR cutoff $T$. In particular, one makes the assumption [26] that the function $\mathcal{G}_{E}$, as a function of the complex variable $\theta$, can be analytically extended from the real segment $(0<\operatorname{Re} \theta<\pi, \operatorname{Im} \theta=0)$ to a domain $\mathcal{D}_{E}$, which also includes the negative imaginary axis $(\operatorname{Re} \theta=0+, \operatorname{Im} \theta<0)$; therefore, the function $\mathcal{G}_{M}$, as a function of the complex variable $\chi$, can be analytically extended from the positive real axis $(\operatorname{Re} \chi>0, \operatorname{Im} \chi=0+)$ to a domain $\mathcal{D}_{M}=\left\{\chi \in \mathbb{C} \mid-i \chi \in \mathcal{D}_{E}\right\}$, which also includes the imaginary segment $(\operatorname{Re} \chi=0,0<\operatorname{Im} \chi<\pi)$. The validity of this assumption is confirmed by explicit calculations in perturbation theory [14, 17, 5]. The domains $\mathcal{D}_{E}$ and $\mathcal{D}_{M}$ are schematically shown in Fig. 2. Denoting with $\overline{\mathcal{G}}_{M}$ and $\overline{\mathcal{G}}_{E}$ such analytic extensions, we then have the following analytic-continuation relations [17, 26]:

$$
\begin{aligned}
\overline{\mathcal{G}}_{E}\left(\theta ; T ; \vec{z}_{\perp} ; 1,2\right)=\overline{\mathcal{G}}_{M}\left(i \theta ;-i T ; \vec{z}_{\perp} ; 1,2\right), \quad \forall \theta \in \mathcal{D}_{E} ; \\
\overline{\mathcal{G}}_{M}\left(\chi ; T ; \vec{z}_{\perp} ; 1,2\right)=\overline{\mathcal{G}}_{E}\left(-i \chi ; i T ; \vec{z}_{\perp} ; 1,2\right), \quad \forall \chi \in \mathcal{D}_{M} .
\end{aligned}
$$

As we have said above, the loop-loop correlation functions (both in the Minkowskian and in the Euclidean theories) are expected to be IR-finite quantities, i.e., to have finite limits when $T \rightarrow \infty$, differently from what happens in the case of Wilson lines. One can then define the following loop-loop correlation functions with the IR cutoff removed:

$$
\begin{aligned}
\mathcal{C}_{M}\left(\chi ; \vec{z}_{\perp} ; 1,2\right) & \equiv \lim _{T \rightarrow \infty} \mathcal{G}_{M}\left(\chi ; T ; \vec{z}_{\perp} ; 1,2\right), \\
\mathcal{C}_{E}\left(\theta ; \vec{z}_{\perp} ; 1,2\right) & \equiv \lim _{T \rightarrow \infty} \mathcal{G}_{E}\left(\theta ; T ; \vec{z}_{\perp} ; 1,2\right) .
\end{aligned}
$$

It has been proved in Ref. [17] that, under certain analyticity conditions in the complex variable $T$ [conditions which are also sufficient to make the relations (2.12) meaningful], the two quantities (2.13), obtained after the removal of the IR cutoff $(T \rightarrow \infty)$, are still

\footnotetext{
${ }^{\dagger}$ We note here that, exploiting the invariance of the theory under charge conjugation $(C)$, one can show that the correlation function $\mathcal{G}_{E}$, as well as the quantity $\mathcal{C}_{E}$ to be defined below in Eq. (2.13), is a real quantity (differently from the corresponding Minkowskian correlator). In fact, under a $C$ transformation, $A_{E \mu} \rightarrow A_{E \mu}^{(c)}=-A_{E \mu}^{t}=-A_{E \mu}^{*}$, the integration measure $\left[d A_{E}\right]$, the gauge-field action $S_{E}$ and the fermion matrix determinant $\operatorname{det} \mathcal{Q}_{E}\left[A_{E}\right]$ (which are real quantities) are invariant, while a Wilson loop $\widetilde{\mathcal{W}}$ goes into its complex conjugate $\widetilde{\mathcal{W}}^{*}$.
} 
connected by the usual analytic continuation in the angular variables only:

$$
\begin{aligned}
\overline{\mathcal{C}}_{E}\left(\theta ; \vec{z}_{\perp} ; 1,2\right) & =\overline{\mathcal{C}}_{M}\left(i \theta ; \vec{z}_{\perp} ; 1,2\right), & & \forall \theta \in \mathcal{D}_{E} ; \\
\overline{\mathcal{C}}_{M}\left(\chi ; \vec{z}_{\perp} ; 1,2\right) & =\overline{\mathcal{C}}_{E}\left(-i \chi ; \vec{z}_{\perp} ; 1,2\right), & & \forall \chi \in \mathcal{D}_{M} .
\end{aligned}
$$

This is a highly non-trivial result, whose general validity is discussed in Ref. [17]. The validity of the relation (2.14) for the loop-loop correlators in QCD has been also verified in Ref. [5] by an explicit calculation up to the order $\mathcal{O}\left(g^{6}\right)$ in perturbation theory. However we want to stress that the analytic continuation (2.12) or (2.14) is expected to be an exact result, i.e., not restricted to some order in perturbation theory or to some other approximation, and is valid both for the Abelian and the non-Abelian cases.

It has also been recently shown in Refs. [26, 23] that the analytic-continuation relations (2.12) allow us to deduce non-trivial properties of the Euclidean correlator $\mathcal{G}_{E}$ under the exchange $\theta \rightarrow \pi-\theta$ and of the Minkowskian correlator $\mathcal{G}_{M}$ under the exchange $\chi \rightarrow i \pi-\chi$, corresponding to the exchange from a loop-loop correlator to a loop-antiloop correlator, where an antiloop is obtained from a given loop by exchanging the quark and the antiquark trajectories:

$$
\begin{aligned}
\mathcal{G}_{E}\left(\pi-\theta ; T ; \vec{z}_{\perp} ; 1,2\right)=\mathcal{G}_{E}\left(\theta ; T ; \vec{z}_{\perp} ; 1, \overline{2}\right)=\mathcal{G}_{E}\left(\theta ; T ; \vec{z}_{\perp} ; \overline{1}, 2\right), & \forall \theta \in(0, \pi) \\
\overline{\mathcal{G}}_{M}\left(i \pi-\chi ; T ; \vec{z}_{\perp} ; 1,2\right)=\mathcal{G}_{M}\left(\chi ; T ; \vec{z}_{\perp} ; 1, \overline{2}\right)=\mathcal{G}_{M}\left(\chi ; T ; \vec{z}_{\perp} ; \overline{1}, 2\right), & \forall \chi \in \mathbb{R}^{+},
\end{aligned}
$$

where the arguments " $\overline{1}$ " and " $\overline{2}$ " stand for " $-\vec{R}_{1 \perp}, 1-f_{1}$ " and " $-\vec{R}_{2 \perp}, 1-f_{2}$ " respectively. These two relations are known as crossing-symmetry relations for loop-loop correlators. As they are valid for every value of the IR cutoff $T$, completely analogous relations also hold for the loop-loop correlation functions $\mathcal{C}_{M}$ and $\mathcal{C}_{E}$ with the IR cutoff removed $(T \rightarrow \infty)$, defined in Eq. (2.13)

$$
\begin{array}{rlrl}
\mathcal{C}_{E}\left(\pi-\theta ; \vec{z}_{\perp} ; 1,2\right) & =\mathcal{C}_{E}\left(\theta ; \vec{z}_{\perp} ; 1, \overline{2}\right)=\mathcal{C}_{E}\left(\theta ; \vec{z}_{\perp} ; \overline{1}, 2\right), & & \forall \theta \in(0, \pi) ; \\
\overline{\mathcal{C}}_{M}\left(i \pi-\chi ; \vec{z}_{\perp} ; 1,2\right) & =\mathcal{C}_{M}\left(\chi ; \vec{z}_{\perp} ; 1, \overline{2}\right)=\mathcal{C}_{M}\left(\chi ; \vec{z}_{\perp} ; \overline{1}, 2\right), & \forall \chi \in \mathbb{R}^{+} .
\end{array}
$$

Taking into account the aforementioned analytic-continuation relations, we can rewrite Eq. (2.8) in terms of the Euclidean correlation function $\mathcal{C}_{E}$ as

$$
\begin{aligned}
\mathcal{M}_{(h h)}(s, t)= & -i 2 s \int d^{2} \vec{R}_{1 \perp} \int_{0}^{1} d f_{1}\left|\psi_{1}\left(\vec{R}_{1 \perp}, f_{1}\right)\right|^{2} \int d^{2} \vec{R}_{2 \perp} \int_{0}^{1} d f_{2}\left|\psi_{2}\left(\vec{R}_{2 \perp}, f_{2}\right)\right|^{2} \\
& \times \int d^{2} \vec{z}_{\perp} e^{i \vec{q}_{\perp} \cdot \vec{z}_{\perp}} \mathcal{C}_{E}\left(\theta \rightarrow-i \log \left(\frac{s}{m^{2}}\right) ; \vec{z}_{\perp} ; \vec{R}_{1 \perp}, f_{1}, \vec{R}_{2 \perp}, f_{2}\right) .
\end{aligned}
$$


By virtue of the optical theorem, Eq. (1.2), the total cross section is then given by the expression

$$
\begin{aligned}
\sigma_{\text {tot }}^{(h h)}(s) \underset{s \rightarrow \infty}{\sim}-2 \int & d^{2} \vec{R}_{1 \perp} \int_{0}^{1} d f_{1}\left|\psi_{1}\left(\vec{R}_{1 \perp}, f_{1}\right)\right|^{2} \int d^{2} \vec{R}_{2 \perp} \int_{0}^{1} d f_{2}\left|\psi_{2}\left(\vec{R}_{2 \perp}, f_{2}\right)\right|^{2} \\
& \times \int d^{2} z_{\perp} \operatorname{Re} \mathcal{C}_{E}\left(\theta \rightarrow-i \log \left(\frac{s}{m^{2}}\right) ; \vec{z}_{\perp} ; \vec{R}_{1 \perp}, f_{1}, \vec{R}_{2 \perp}, f_{2}\right) .
\end{aligned}
$$

If one chooses hadron wave functions invariant under rotations and under the exchange $f_{i} \rightarrow 1-f_{i}$ (see Refs. [12, 13] and also [1], §8.6, and references therein), the correlation function $\mathcal{C}_{E}$ in Eqs. (2.17) and (2.18) can be substituted (without changing the result) with the following averaged correlation function:

$$
\begin{aligned}
& \mathcal{C}_{E}^{\text {ave }}\left(\theta ; \vec{z}_{\perp} ;\left|\vec{R}_{1 \perp}\right|, f_{1},\left|\vec{R}_{2 \perp}\right|, f_{2}\right) \equiv \int d \hat{R}_{1 \perp} \int d \hat{R}_{2 \perp} \\
& \quad \times \frac{1}{4}\left\{\mathcal{C}_{E}\left(\theta ; \vec{z}_{\perp} ; \vec{R}_{1 \perp}, f_{1}, \vec{R}_{2 \perp}, f_{2}\right)+\mathcal{C}_{E}\left(\theta ; \vec{z}_{\perp} ; \vec{R}_{1 \perp}, 1-f_{1}, \vec{R}_{2 \perp}, f_{2}\right)\right. \\
&\left.\quad+\mathcal{C}_{E}\left(\theta ; \vec{z}_{\perp} ; \vec{R}_{1 \perp}, f_{1}, \vec{R}_{2 \perp}, 1-f_{2}\right)+\mathcal{C}_{E}\left(\theta ; \vec{z}_{\perp} ; \vec{R}_{1 \perp}, 1-f_{1}, \vec{R}_{2 \perp}, 1-f_{2}\right)\right\},
\end{aligned}
$$

where $\int d \hat{R}_{i \perp}$ stands for integration over the orientations of $\vec{R}_{i \perp}$. We note here that, as a consequence of the crossing-symmetry relations Eq. (2.16), the function $\mathcal{C}_{E}^{\text {ave }}$ is automatically crossing-symmetric, i.e., $\mathcal{C}_{E}^{\text {ave }}(\pi-\theta ; \ldots)=\mathcal{C}_{E}^{\text {ave }}(\theta ; \ldots)$ for fixed values of the other variables.

In the following, if not specified otherwise, we will take for simplicity the longitudinalmomentum fractions $f_{1}$ and $f_{2}$ of the two quarks in the two dipoles (and, therefore, also the longitudinal-momentum fractions $1-f_{1}$ and $1-f_{2}$ of the two antiquarks in the two dipoles) to be fixed to $1 / 2$ : this is known to be a good approximation for hadron-hadron interactions (see Refs. [1, 12] and references therein). We will also adopt the notation $\mathcal{G}_{E}\left(\theta ; T ; \vec{z}_{\perp} ; \vec{R}_{1 \perp}, \vec{R}_{2 \perp}\right) \equiv \mathcal{G}_{E}\left(\theta ; T ; \vec{z}_{\perp} ; \vec{R}_{1 \perp}, f_{1}=\frac{1}{2}, \vec{R}_{2 \perp}, f_{2}=\frac{1}{2}\right)$, and similarly for $\mathcal{C}_{E}$ and $\mathcal{C}_{E}^{\text {ave }}$. Note that in this case the quantity $\mathcal{C}_{E}^{\text {ave }}$ reduces to the average over the transverse orientations only:

$$
\mathcal{C}_{E}^{\text {ave }}\left(\theta ; \vec{z}_{\perp} ;\left|\vec{R}_{1 \perp}\right|,\left|\vec{R}_{2 \perp}\right|\right)=\int d \hat{R}_{1 \perp} \int d \hat{R}_{2 \perp} \mathcal{C}_{E}\left(\theta ; \vec{z}_{\perp} ; \vec{R}_{1 \perp}, \vec{R}_{2 \perp}\right)
$$

In the next section we will show how it is possible to calculate these Euclidean correlation functions in LGT and discuss the main technical difficulties of this approach. 


\section{Loop-loop correlators on the lattice}

Being a gauge-invariant quantity, the Wilson-loop correlation function $\mathcal{G}_{E}$ is a natural candidate for a lattice computation; nevertheless, a number of complications arise because of the explicit breaking of $O(4)$ invariance on a lattice. The major complication is due to the limited number of possible orientations of rectangular loops on the lattice. As straight lines on a hypercubic lattice can be either parallel or orthogonal, the values of $\theta$ directly accessible are limited to $0^{\circ}, 90^{\circ}$ and $180^{\circ}$. To cover a significantly large set of angles, we then have to make use of off-axis (and so non planar) Wilson loops, thus introducing in our approach another approximation that should be carefully discussed.

The loops involved in the calculation of $\mathcal{G}_{E}$ have one side (from now on the longitudinal side) in the $\left(x_{E 1}, x_{E 4}\right)$ (longitudinal) plane and the other (transverse side) in the $\left(x_{E 2}, x_{E 3}\right)$ (transverse) plane and their centers are separated in the transverse plane, so that the problem of reproducing the loop configuration consists effectively of two distinct twodimensional problems. It is reasonable to evaluate the loop sides on the lattice paths that minimise the distance from the true, continuum paths, in order to stay as "close" as possible to the continuum limit, adopting essentially the same strategy as in computer graphics when drawing straight lines on a screen (see Fig. 3); such "minimal-distance paths" can be found in a very efficient way by means of the well-known Bresenham algorithm [27, which has already been used in lattice calculations (see e.g. [28], where it is also generalised to the three-dimensional case). To every continuum straight path having as endpoints two coplanar lattice points we can then associate unambiguously a Wilson line on the lattice by means of the minimal-distance prescription* and then build with them the Wilson loops we are interested in. Such loops are identified by the position of the center and by two two-dimensional lattice vectors; we thus define $\widetilde{\mathcal{W}}_{L}\left(\vec{l}_{\|} ; \vec{r}_{\perp} ; n\right)$ to be the lattice Wilson loop evaluated on the minimal path that approximates the rectangle having as corners the lattice points $n-l / 2-r / 2, n+l / 2-r / 2, n+l / 2+r / 2$ and $n-l / 2+r / 2$, where $n, l$ and $r$ are vectors in lattice units, $n$ is the position of the center and $\vec{l}_{\|}=\left(l_{1}, l_{4}\right), l_{2}=l_{3}=0, \vec{r}_{\perp}=\left(r_{2}, r_{3}\right), r_{1}=r_{4}=0$.

\footnotetext{
*For lines inclined at $45^{\circ}$ with respect to an axis a certain ambiguity remains, but we can average over equivalent paths.

$\dagger$ The components of $l$ and $r$ must be integers. Note that the center of the loop may not lie on a lattice point.
} 
On the lattice we then define the correlator

$$
\mathcal{G}_{L}\left(\vec{l}_{1 \|}, \vec{l}_{2 \|} ; \vec{d}_{\perp} ; \vec{r}_{1 \perp}, \vec{r}_{2 \perp}\right) \equiv \frac{\left\langle\widetilde{\mathcal{W}}_{L}\left(\vec{l}_{1 \|} ; \vec{r}_{1 \perp} ; d\right) \widetilde{\mathcal{W}}_{L}\left(\vec{l}_{2 \|} ; \vec{r}_{2 \perp} ; 0\right)\right\rangle}{\left\langle\widetilde{\mathcal{W}}_{L}\left(\vec{l}_{1 \|} ; \vec{r}_{1 \perp} ; d\right)\right\rangle\left\langle\widetilde{\mathcal{W}}_{L}\left(\vec{l}_{2 \|} ; \vec{r}_{2 \perp} ; 0\right)\right\rangle}-1
$$

where $d=\left(0, \vec{d}_{\perp}, 0\right), \vec{d}_{\perp}=\left(d_{2}, d_{3}\right)$. As (full) $O(4)$ invariance is broken, this correlator depends explicitly on the (two-dimensional) lattice vectors $\vec{l}_{i \|}, \vec{r}_{i \perp}(i=1,2)$ and $\vec{d}_{\perp}$ rather than on their scalar products: indeed, for a given relative orientation we can find different realisations in terms of lattice vectors, generally inequivalent as they involve different Wilson-loop operators. Anyway, as rotation invariance is restored in the continuum limit, we expect

$$
\mathcal{G}_{L}\left(\vec{l}_{1 \|}, \vec{l}_{2 \|} ; \vec{d}_{\perp} ; \vec{r}_{1 \perp}, \vec{r}_{2 \perp}\right) \underset{a \rightarrow 0}{\simeq} \mathcal{G}_{E}\left(\theta ; T_{1}=a L_{1} / 2, T_{2}=a L_{2} / 2 ; a \vec{d}_{\perp} ; a \vec{r}_{1 \perp}, a \vec{r}_{2 \perp}\right),
$$

where $L_{i} \equiv\left|\vec{l}_{i \|}\right|$ are what we define to be the lengths of the longitudinal sides of the loops in lattice units (from now on, "lengths"), and $\vec{l}_{1 \|} \cdot \vec{l}_{2 \|} \equiv L_{1} L_{2} \cos \theta$. As it is impossible, in general, to have $L_{1}=L_{2}$, we have relaxed this condition and have considered in Eq. (3.2) the correlation function with two IR cutoffs $T_{1}$ and $T_{2}$,

$$
\mathcal{G}_{E}\left(\theta ; T_{1}, T_{2} ; \vec{z}_{\perp} ; \vec{R}_{1 \perp}, \vec{R}_{2 \perp}\right) \equiv \frac{\left\langle\widetilde{\mathcal{W}}_{1}^{\left(T_{1}\right)} \widetilde{\mathcal{W}}_{2}^{\left(T_{2}\right)}\right\rangle}{\left\langle\widetilde{\mathcal{W}}_{1}^{\left(T_{1}\right)}\right\rangle\left\langle\widetilde{\mathcal{W}}_{2}^{\left(T_{2}\right)}\right\rangle}-1
$$

where with a small abuse of notation we have kept the same notation for the correlation function as in Eq. (2.11). As already pointed out in Section 2, the correlator $\mathcal{G}_{E}$ is expected to be an IR-finite quantity, so that we can regularise it considering loops with different lengths $T_{1}$ and $T_{2}$ and taking the limits $T_{1}, T_{2} \rightarrow \infty$ independently, obtaining the same function $\mathcal{C}_{E}$ defined in Eq. (2.13).

On top of these field theoretical complications we have to face the numerical difficulty (or even feasibility) of a lattice "measurement" of the relevant correlation function of Wilson loops. The Wilson-loop expectation value is known to obey an "area law", i.e., to vanish exponentially with its area for large areas; moreover, in the 't Hooft large- $N_{c}$ expansion with $g^{2} N_{c}$ kept constant such correlators are known to factorise to leading order, thus giving

$$
\mathcal{G}_{E}=\frac{\left\langle\widetilde{\mathcal{W}}_{1}^{(T)} \widetilde{\mathcal{W}}_{2}^{(T)}\right\rangle}{\left\langle\widetilde{\mathcal{W}}_{1}^{(T)}\right\rangle\left\langle\widetilde{\mathcal{W}}_{2}^{(T)}\right\rangle}-1=\frac{\left\langle\widetilde{\mathcal{W}}_{1}^{(T)}\right\rangle\left\langle\widetilde{\mathcal{W}}_{2}^{(T)}\right\rangle+\mathcal{O}\left(\frac{1}{N_{c}^{2}}\right)}{\left\langle\widetilde{\mathcal{W}}_{1}^{(T)}\right\rangle\left\langle\widetilde{\mathcal{W}}_{2}^{(T)}\right\rangle}-1=\mathcal{O}\left(\frac{1}{N_{c}^{2}}\right) .
$$


We then expect $\mathcal{G}_{E}$ to be a small quantity, obtained from the ratio of two exponentially decreasing quantities as $T$ becomes large. Moreover, general considerations make plausible a behaviour of the kind $\mathcal{G}_{E} \sim e^{-\gamma\left|\vec{z}_{\perp}\right|}$ at large separations, and thus we expect the noise to overcome the signal after a few lattice spacings.

We can try to maximise the information obtained from each thermalised configuration, in order to reduce the statistical noise, by exploiting the symmetries of the lattice. It is easy to see that the chosen prescription for the construction of loops is consistent with lattice rotations and reflections [i.e., the cubic subgroup of $O(4)$ ], in the following sense. One can perform a cubic transformation on a Wilson loop (in the continuum), and then construct its lattice approximation with the given prescription, or alternatively construct first the lattice approximation and then perform the same cubic transformation (this time on the lattice), and in both cases one would obtain the same result. We can then average over cubic transformations of the whole loop configuration and, imposing periodic boundary conditions, we can average over lattice translations as well; to further clarify the numerical signal we can also use the identities

$$
\begin{aligned}
& \mathcal{G}_{L}\left(\vec{l}_{1 \|}, \vec{l}_{2 \|} ; \vec{d}_{\perp} ; \vec{r}_{1 \perp}, \vec{r}_{2 \perp}\right)=\mathcal{G}_{L}\left(\vec{l}_{1 \|}, \vec{l}_{2 \|} ;-\vec{d}_{\perp} ;-\vec{r}_{1 \perp},-\vec{r}_{2 \perp}\right) \\
& =\mathcal{G}_{L}\left(\vec{l}_{1 \|}, \vec{l}_{2 \|} ;-\vec{d}_{\perp} ; \vec{r}_{1 \perp}, \vec{r}_{2 \perp}\right)=\mathcal{G}_{L}\left(\vec{l}_{1 \|}, \vec{l}_{2 \|} ; \vec{d}_{\perp} ;-\vec{r}_{1 \perp},-\vec{r}_{2 \perp}\right)
\end{aligned}
$$

which can be proved using invariance under cubic symmetry and the trivial fact that

$$
\widetilde{\mathcal{W}}_{L}\left(\vec{l}_{\|} ;-\vec{r}_{\perp} ; n\right)=\widetilde{\mathcal{W}}_{L}\left(-\vec{l}_{\|} ; \vec{r}_{\perp} ; n\right)
$$

\section{Numerical results}

We have performed a Monte Carlo calculation of the correlation function $\mathcal{G}_{L}$ of two Wilson loops for several values of the relative angle, various lengths and different configurations in the transverse plane, on a $16^{4}$ hypercubic lattice with periodic boundary conditions. The link configurations were generated by means of a mixture of (pseudo)heatbath [29, 30, 31] and overrelaxation steps [32] with the usual Wilson action for $S U(3)$ pure-gauge theory [33, also known in the literature as the quenched approximation of 
QCD, which consists in neglecting dynamical fermion loops by setting the fermion matrix determinant to a constant. Even though, of course, we cannot exclude that the inclusion of dynamical-fermion effects, via the fermion-matrix determinant, could introduce new features in the data, in this paper (which, we want to stress, is the first to approach this problem from the point of view of lattice QCD) we have preferred to make the easiest and most convenient choice, that gives us the possibility of collecting large statistics (also considering the various difficulties in measuring the correlation functions $\mathcal{G}_{L}$, as explained in the previous section).

We have measured the correlation functions $\left\langle\widetilde{\mathcal{W}}_{L 1} \widetilde{\mathcal{W}}_{L 2}\right\rangle$ and the loop expectation values $\left\langle\widetilde{\mathcal{W}}_{L 1}\right\rangle$ and $\left\langle\widetilde{\mathcal{W}}_{L 2}\right\rangle$, with $\widetilde{\mathcal{W}}_{L 1} \equiv \widetilde{\mathcal{W}}_{L}\left(\vec{l}_{1 \|} ; \vec{r}_{1 \perp} ; d\right)$ and $\widetilde{\mathcal{W}}_{L 2} \equiv \widetilde{\mathcal{W}}_{L}\left(\vec{l}_{2 \|} ; \vec{r}_{2 \perp} ; 0\right)$, on 30000 thermalised configurations at $\beta \equiv 6 / g^{2}=6.0$. As it is well known, the lattice spacing $a$ is related to the bare coupling constant $g$ (i.e., to $\beta$ ) through the renormalisation group equation. The lattice scale, i.e., the value of $a$ in physical units, is determined from the physical value of some relevant (dimensionful) observable like the string tension or the static $q \bar{q}$ force at some fixed distance ("Sommer scale") (see e.g. Ref. [34] and references therein): in our case one finds that $a(\beta=6.0) \simeq 0.1 \mathrm{fm}$. The choice of $\beta=6.0$ on a $16^{4}$ lattice is made in order to stay within the so-called "scaling window": in this sense we are relying in an indirect way on the validity of the relation (3.2) between Wilson-loop correlation functions on the lattice and in the continuum (and therefore we shall use the notation $\mathcal{G}_{E} / \mathcal{C}_{E}$ of the continuum in all the figures reporting our lattice data). An explicit test of scaling in our case is more difficult, as one has to keep a large number of length scales under control while varying the lattice spacing. A possibility could be to integrate over the distance and the sizes of the loops and then study the scaling properties of the resulting quantity, although this seems to be a very hard task.

To keep the corrections due to $O(4)$ invariance breaking as small as possible, we have kept one of the two loops on-axis and we have only tilted the other one as shown in Fig. 4: the on-axis loop $\widetilde{\mathcal{W}}_{L 1}$ is taken to be parallel to the $x_{E 1}$ axis, $\vec{l}_{1 \|}=\left(L_{1}, 0\right)$, and of length $L_{1}=6,8$. We have used two sets of off-axis loops $\widetilde{\mathcal{W}}_{L 2}$ tilted at $\tan ^{-1}(1 / 2) \simeq 26.565^{\circ}$ and $45^{\circ}$ with respect to one of the longitudinal axes; the corresponding lattice vectors $\vec{l}_{2 \|}$ are listed in Table 1, together with their length and the angle formed with $\vec{l}_{1 \|}$. We have used loops with transverse size $\left|\vec{r}_{1 \perp}\right|=\left|\vec{r}_{2 \perp}\right|=1$ in lattice units; the loop configurations

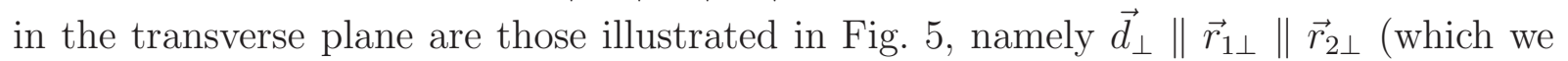
call "zzz") and $\vec{d}_{\perp} \perp \vec{r}_{1 \perp} \| \vec{r}_{2 \perp}$ ("zyy"). As explained in Section 2, it is interesting to also measure the orientation-averaged quantity ("ave") defined in Eq. (2.20). The lattice 
version of this equation is easily recovered for even (integer) values of the transverse sizes; in our particular case, $\left|\vec{r}_{i \perp}\right|=1$, we have to use a sort of "smearing" procedure, averaging nearby loops as depicted in Fig. 5. Note that in doing so we are actually averaging over the orientations and over the values $f_{i}=0,1(i=1,2)$ of the longitudinal-momentum fractions, according to Eq. (2.19).

As explained in Section 2, we are interested in the $T \rightarrow \infty$ limit and so we have to somehow perform it on the lattice. In practice, we have to look for a plateau of the correlation function plotted against the loop lengths $L_{1}$ and $L_{2}$ : in Fig. 6 we show the dependence of the correlator on the length $L_{1}=L_{2}=L$ of the loops at $\theta=90^{\circ}$. Of course, on a $16^{4}$ lattice it is difficult to have a sufficiently long loop while at the same time avoiding finite size effects and at best we can push the calculation up to $L=8$; nevertheless, a plateau seems to have been practically reached at about $L=L_{\mathrm{pl}} \simeq 6-8$. As $\theta$ varies from $90^{\circ}$ towards $0^{\circ}$ or $180^{\circ}$, we expect $L_{\mathrm{pl}}$ to grow*; however, the plots in Figs. [7] 9 show that the correlation function is already quite stable against variations of the loop lengths at $L_{1}, L_{2} \simeq 8$ (at least for $\theta$ not too close to $0^{\circ}$ or $180^{\circ}$ ) and so we can take the data for the largest loops available as a reasonable approximation of $\mathcal{C}_{L}$, defined as the asymptotic value of $\mathcal{G}_{L}$ as $L_{1}, L_{2} \rightarrow \infty$. We estimate the uncertainty in $\mathcal{C}_{L}$ due to this approximation from the variation of $\mathcal{G}_{L}$ with the lenghts $L_{1}, L_{2}$ as (using the notation introduced in Table 1)

$$
\begin{aligned}
\left(\delta \mathcal{C}_{L}\right)_{\text {syst }}= & \frac{1}{2}\left\{\mid \mathcal{G}_{L}\left(L_{1}=8, \text { set }=2\right) \quad-\mathcal{G}_{L}\left(L_{1}=8, \text { set }=1\right) \mid\right. \\
& \left.+\mid \mathcal{G}_{L}\left(L_{1}=8, \text { set }=2\right) \quad-\mathcal{G}_{L}\left(L_{1}=6, \text { set }=2\right) \mid\right\} .
\end{aligned}
$$

The errors shown in Figs. 7 9 are the statistical ones only; in all the other figures we show the total error obtained adding (in quadrature) the statistical and the "systematic" errors defined above.

As already noticed above, the data are less stable for $\theta$ near $0^{\circ}$ and $180^{\circ}$ : this is a consequence of the relation between the correlation function $\mathcal{G}_{E}$ and the static dipoledipole potential $V_{12}$ [35],

$$
\mathcal{G}_{E}\left(\theta=0 ; T ; \vec{z}_{\perp} ; \vec{R}_{1 \perp}, \vec{R}_{2 \perp}\right) \underset{T \rightarrow \infty}{\simeq} \exp \left[-2 T V_{12}\left(\vec{z}_{\perp}, \vec{R}_{1 \perp}, \vec{R}_{2 \perp}\right)\right]-1
$$

(note that we have set again $T_{1}=T_{2}=T$ ), from which we expect $\mathcal{G}_{E}$ to diverge at $\theta=0^{\circ}$;

\footnotetext{
${ }^{*}$ Indeed, $L_{\mathrm{pl}}$ blows up at $0^{\circ}, 180^{\circ}$ due to the relation between the correlation function and the static dipole-dipole potential to be discussed below, see Eq. (4.2).
} 


\begin{tabular}{lr|rrrrrrr} 
& $\theta$ & $26.565^{\circ}$ & $45^{\circ}$ & $63.435^{\circ}$ & $90^{\circ}$ & $116.565^{\circ}$ & $135^{\circ}$ & $153.435^{\circ}$ \\
\hline$\vec{l}_{2 \|}$ & set 1 & & & & & & & \\
& set 2 & $(4,2)$ & $(4,4)$ & $(2,4)$ & $\begin{array}{c}(0,6) \\
(6,6)\end{array}$ & $\begin{array}{r}(-2,4) \\
(4,8)\end{array}$ & $\begin{array}{r}(-4,4) \\
(0,8)\end{array}$ & $\begin{array}{c}(-4,4) \\
(-8,8)\end{array}$ \\
\hline & & & & & & & & \\
$L_{2}$ & set 1 & $2 \sqrt{5}$ & $4 \sqrt{2}$ & $2 \sqrt{5}$ & 6 & $2 \sqrt{5}$ & $4 \sqrt{2}$ & $2 \sqrt{5}$ \\
& set 2 & $4 \sqrt{5}$ & $6 \sqrt{2}$ & $4 \sqrt{5}$ & 8 & $4 \sqrt{5}$ & $6 \sqrt{2}$ & $4 \sqrt{5}$ \\
\hline
\end{tabular}

Table 1: Longitudinal vector $\vec{l}_{2 \|}$ and length $L_{2}$ for the various angles and for the two sets of off-axis loops $\widetilde{\mathcal{W}}_{L 2}$.

by virtue of the crossing-symmetry relations (2.15), a similar singularity is also expected at $\theta=180^{\circ}$ [26]. In the following we will consider only $\theta \neq 0^{\circ}, 180^{\circ}$.

We have considered the values $d=0,1,2$ for the distance between the centers of the loops. As expected (see the previous Section), the correlation functions vanish rapidly as $d$ increases, as can be seen in Fig. 10, thus making the calculation with our simple "brute force" approach very difficult at larger distances.

From now on we will discuss the issue of the angular dependence of the correlation function. As already pointed out in the Introduction, numerical simulations of LGT can provide the Euclidean correlation function only for a finite set of $\theta$-values, and so its analytic properties cannot be directly attained; nevertheless, they are first-principles calculations that give us (inside the errors) the true QCD expectation for this quantity. Approximate analytic calculations of this same function then have to be compared with the lattice data, in order to test the goodness of the approximations involved. The Euclidean correlation functions we are interested in have been evaluated in the SVM [18], in the ILM [19] and using the AdS/CFT correspondence (in the "conformal" 20] and "nonconformal" cases [21]). The SVM gives a well-defined quantitative prediction, that can be tested numerically against our data; in the ILM and conformal-AdS/CFT cases we have qualitative knowledge of the functional dependence of the correlation functions on the angle $\theta$ and we can test at least the goodness of the functional form with a fit to the lattice data; in the non-conformal-AdS/CFT case the explicit $\theta$-dependence is unknown and we are unable to make any comparison with our data.

In the SVM [18] the Wilson-loop correlation function in $N_{c}$-colour QCD $\left(N_{c}=3\right.$ in 
our case) is given by the expression

$$
\begin{aligned}
\mathcal{C}_{E}^{(\mathrm{SVM})}(\theta) & =\left(\frac{N_{c}+1}{2 N_{c}}\right) \exp \left[-\left(\frac{N_{c}-1}{2 N_{c}}\right) K_{\mathrm{SVM}} \cot \theta\right] \\
& +\left(\frac{N_{c}-1}{2 N_{c}}\right) \exp \left[\left(\frac{N_{c}+1}{2 N_{c}}\right) K_{\mathrm{SVM}} \cot \theta\right]-1,
\end{aligned}
$$

where $K_{\mathrm{SVM}}$ is a function of $\vec{z}_{\perp}, \vec{R}_{1 \perp}$ and $\vec{R}_{2 \perp}$ only, whose precise expression, which we have used to numerically evaluate the correlator (4.3) in the relevant cases, is given in Ref. [18. The comparison of the SVM prediction for $\mathcal{C}_{E}$ with our data is shown in Figs. 11,13, in the "ave" case the comparison is made with $\mathcal{C}^{\text {ave }}$ as defined in Eq. (2.19), with $f_{1}=f_{2}=0$ (see discussion above). Although in some cases the agreement is quite good, at least in the shape of the curve and in the order of magnitude, in general it is far from being satisfactory; in particular, in the "zyy" case for $d \neq 0$ the SVM prediction is orders of magnitude smaller than the lattice results. One can also go the other way round, namely try to determine $K_{\mathrm{SVM}}$ in the " $z z z$ " and " $z y y$ " cases with a one-parameter best-fit to the data: the results are shown in Figs. 1113. In general, the difference between the predicted and the fitted values for $K_{\mathrm{SVM}}$ is positive and in some cases the discrepancy is larger than 20\%, which can be taken as the accuracy level of the model parameters (see Ref. [18]). We note that for $d=0,1$ the main contribution to the value of $K_{\mathrm{SVM}}$, evaluated using the SVM expression given in Ref. [18] (which, we recall, consists of a perturbative plus a nonperturbative component) comes from perturbative effects, while at $d=2$ nonperturbative effects are equal to or greater than the perturbative ones. We have also tried a best-fit with the following simple functional form:

$$
\mathcal{C}_{E}^{(\text {pert })}(\theta)=K_{\text {pert }}(\cot \theta)^{2}
$$

which is exactly what one obtains in leading-order perturbation theory [5, 17, 18]. Notice, however, that the coefficient $K_{\text {pert }}$ in Eq. (4.4) can also receive nonperturbative contributions, as one can see, for example, when expanding the exponentials in the SVM expression (4.3) to first ordent. The values of the chi-squared per degree of freedom $\left(\chi_{\text {d.o.f. }}^{2}\right)$ of the various fits that we have performed are listed in Table 2.

\footnotetext{
${ }^{\dagger}$ Here and in the following formulae we omit the variables other than $\theta$ on which $\mathcal{C}_{E}$ depends.

${ }^{\ddagger}$ This makes sense when $K_{\mathrm{SVM}}$ is small, as it happens for example in the large $-N_{c}$ expansion, where it is $K_{\mathrm{SVM}}=\mathcal{O}\left(\frac{1}{N_{c}}\right)$.
} 
One-instanton effects in the ILM give the following analytic expression for the correlation function [19]:

$$
\mathcal{C}_{E}^{(\mathrm{ILM})}(\theta)=\frac{K_{\mathrm{ILM}}}{\sin \theta} .
$$

Lattice data are not well fitted by such a function, at least at $d=0,1$; at $d=2$ practically all the fits we have tried are good, as one can see in Table 2, but we interpret this as the result of the large data errors. We can largely improve the fits by adding a term proportional to $(\cot \theta)^{2}$,

$$
\mathcal{C}_{E}^{(\mathrm{ILMp})}(\theta)=\frac{K_{\mathrm{ILMp}}}{\sin \theta}+K_{\mathrm{ILMp}}^{\prime}(\cot \theta)^{2},
$$

which describes contributions both from two-instanton [19] and, as we have already said above, from leading-order perturbative effects; in the following, we will refer to Eq. (4.6) as the "ILMp" expression. Differently from the SVM case (and also from the AdS/CFT case to be discussed below), the dependence on $\theta$ is not affected by the average over transverse orientations (and longitudinal-momentum fractions), so it makes sense to try to also fit the "averaged" data with these functional forms. The resulting best-fit functions in the $d=0,1$ cases are plotted in Figs. 14 and 15, As the fit gives $K_{\mathrm{ILMp}}^{\prime} \gg K_{\mathrm{ILMp}}^{2}$, it seems more likely that $K_{\mathrm{ILMp}}^{\prime}$ is dominated by perturbative effects; while $K_{\mathrm{ILMp}}^{\prime}$ is an order of magnitude larger than $K_{\mathrm{ILMp}}$ at $d=0$, suggesting that perturbative effects are dominant, at $d=1$ the two parameters are comparable, and at $d=2 K_{\text {ILMp }}$ becomes larger than $K_{\mathrm{ILMp}}^{\prime}$, suggesting that we are entering the nonperturbative region.

Using the AdS/CFT correspondence, one obtains for the $\mathcal{N}=4$ SYM theory at large $N_{c}$ and large 't Hooft coupling, and at large distances between the loops [20]

$$
\mathcal{C}_{E}^{(\mathrm{AdS} / \mathrm{CFT})}(\theta)=\exp \left\{K_{1} \frac{1}{\sin \theta}+K_{2} \cot \theta+K_{3} \cos \theta \cot \theta\right\}-1
$$

Although, of course, there is apparently no reason for this formula to apply in our case, we nevertheless have tried to fit the lattice data (in the " $z z z$ " and " $z y y$ " cases) also with this functional form; the results are shown in Fig. 16. The fit is not good at $d=0$; it may seem good at $d=1,2$, but we must remember that this is a three-parameter fit, i.e., there are only four degrees of freedom: the ILMp best-fit, which has only two parameters (i.e., five degrees of freedom) achieves a better or comparable $\chi_{\text {d.o.f. }}^{2}$ and, moreover, we 


\begin{tabular}{l|cc|ccc|ccc}
$\chi_{\text {d.o.f. }}^{2}$ & \multicolumn{2}{|c|}{$d=0$} & \multicolumn{3}{c|}{$d=1$} & \multicolumn{3}{c}{$d=2$} \\
& zzz/zyy & ave & $z z z$ & zyy & ave & $z z z$ & $z y y$ & ave \\
\hline \hline SVM & 51 & - & 16 & 12 & - & 1.5 & 2.2 & - \\
pert & 53 & 34 & 16 & 13 & 13 & 1.5 & 2.2 & 4.5 \\
ILM & 114 & 94 & 14 & 15 & 45 & 0.45 & 0.35 & 1.45 \\
ILMp & 20 & 9.4 & 0.54 & 0.92 & 1.8 & 0.13 & 0.12 & 0.19 \\
AdS/CFT & 40 & - & 1 & 0.63 & - & 0.14 & 0.065 & -
\end{tabular}

Table 2: Chi-squared per degree of freedom for a best-fit with the indicated function.

have explicitly tested some modified versions of the SVM expression (4.3) with two and three parameters in the exponents, obtaining smaller values for $\chi_{\text {d.o.f. }}^{2}$.

As we have said in the Introduction, the main motivation in studying soft high-energy scattering is that it can lead to a resolution of the total cross section puzzle, so it is worth discussing what the various models have to say on this point. Using Eq. (2.18) it is easy to see that the SVM, the ILM and the lowest order perturbative expressions give constant cross sections at high energy $\$$, as in these cases the high-energy limit can be carried over under the integral sign, so that the knowledge of the $\theta$-dependence of the correlation function is sufficient to completely determine the high-energy behaviour of total cross sections. However, it may happen that the integrand in Eq. (2.18) has no definite asymptotic behaviour at large $s$, as it happens, e.g., for the AdS/CFT expression (4.7), so that the remaining integrations have to be carried out before taking $s \rightarrow \infty$. Anyway, as the experimentally observed universality suggests that the high-energy behaviour is not affected by the details of the hadron wave functions (and thus by the detailed dependence of the correlation function on $\vec{R}_{i \perp}$ and $f_{i}$ ), we would expect that the high-energy asymptotics could be read off after integrating only over the distance $\left|\vec{z}_{\perp}\right|$ between the loops and this would require the detailed knowledge of the dependence of the correlators on $\left|\vec{z}_{\perp}\right|$. As already noticed above, however, lattice data show that the relevant correlation functions vanish rapidly with the distance: due to our limited numerical knowledge we postpone the detailed discussion of the dependence on the distance and related issues to subsequent works.

As a final and important remark, we note that our data show a clear signal of $C$-odd contributions in dipole-dipole scattering. As shown in Ref. [26] (and briefly recalled in

\footnotetext{
$\S$ Actually, the ILM expression (4.5) for the correlation function (where we have to recall that $K_{\text {ILM }}$ is a real function) results in an exactly zero total cross section, when inserted in Eq. (2.18).
} 
Section 2) the loop-antiloop correlator at angle $\theta$ in the Euclidean theory (or at hyperbolic angle $\chi$ in the Minkowskian theory) can be derived from the corresponding loop-loop correlator by the substitution $\theta \rightarrow \pi-\theta$ (or $\chi \rightarrow i \pi-\chi$ in the Minkowskian theory). Because of this crossing-symmetry relation, it is natural (see Ref. [23]) to decompose the Euclidean correlation function $\mathcal{C}_{E}(\theta)$ as a sum of a crossing-symmetric function $\mathcal{C}_{E}^{+}(\theta)$ and a crossing-antisymmetric function $\mathcal{C}_{E}^{-}(\theta)$,

$$
\mathcal{C}_{E}(\theta)=\mathcal{C}_{E}^{+}(\theta)+\mathcal{C}_{E}^{-}(\theta), \quad \mathcal{C}_{E}^{ \pm}(\theta) \equiv \frac{\mathcal{C}_{E}(\theta) \pm \mathcal{C}_{E}(\pi-\theta)}{2}
$$

Upon analytic continuation from the Euclidean to the Minkowskian theory, using Eq. (2.16), one can show that they are related respectively to pomeron (i.e., $C=+1$ ) and odderon (i.e., $C=-1$ ) exchanges in the dipole-dipole scattering amplitude. A small but non-zero crossing-antisymmetric component $\mathcal{C}_{E}^{-}$is present in our data, thus signalling the presence of odderon contributions to the loop-loop correlation functions and in turn to the dipole-dipole scattering amplitudes. (Such contributions are absent in meson-meson elastic scattering [36, 23], since in this case the relevant correlation function $C_{E}^{\text {ave }}$ is automatically crossing-symmetric, as already noticed at the end of Section 2). In Fig. 17 we show the crossing-antisymmetric part $\mathcal{C}_{E}^{-}$of the "zzz/zyy" data at $d=0$, together with the corresponding SVM prediction.

\section{Conclusions and outlook}

In this paper the problem of high-energy hadron-hadron (dipole-dipole) scattering has been addressed (for the first time) from the point of view of lattice QCD. We have performed Monte Carlo numerical computations of Euclidean Wilson-loop correlation functions in $S U(3)$ pure-gauge LGT. The energy dependence of soft scattering amplitudes at high energies is encoded in the dependence of these correlation functions on the relative angle $\theta$ between the loops and can be reconstructed after the analytic continuation $\theta \rightarrow$ $-i \log s / m^{2}$.

In this paper we have focused on the study of the angular dependence of the Euclidean correlation function. An interesting and important feature that we have found is the presence of an asymmetry with respect to $\theta=\pi / 2$ in the plot of the Euclidean correlation 
function against the relative angle: this (upon analytic continuation from Euclidean to Minkowskian theory) is a signal of the presence of $C$-odd contributions in dipole-dipole scattering, i.e., a signal of odderon exchange between the dipoles. Even though these $C_{-}$ odd contributions are averaged to zero in meson-meson scattering (at least in our model, as long as the squared meson wave functions satisfy some reasonable symmetry properties in their dependence on the dipole orientations and on the longitudinal-momentum fractions), they might play a non-trivial role in more general hadron-hadron processes in which baryons and antibaryons are also involved.

Although we cannot determine exactly the angular dependence from a finite set of numerical values, we can nevertheless compare the prediction of any given model with the lattice data by direct numerical comparison, if the model is quantitative, or by testing the given functional form with a best-fit to the data, if at least the $\theta$-dependence is known; in this way we can discriminate between various proposals and thus check the goodness of the approximations involved in the specific models. [One can of course also try to fit the data with some given arbitrary functions and then look at the results of the best-fits, but in accepting or rejecting a given function one must also take into account physical arguments, as two fitting functions can differ numerically by a small amount and nevertheless have different analytic structures that can result in completely different (and sometimes physically unacceptable!) high-energy behaviours after analytic continuation to Minkowski space-time.]

The comparison of our data with the existing analytic calculations is not, generally speaking, fully satisfactory.

- The SVM prediction (4.3) agrees with our lattice data in a few cases, at least in the shape and in the order of magnitude, but, in general, it is far from being satisfactory. More or less the same conclusion is reached if one instead performs a one-parameter best-fit with the given expression. This suggests that corrections to the SVM are in order, which could be relevant when deriving the high-energy behaviour of the scattering amplitudes, upon analytic continuation to Minkowski space-time.

- We have then tried best-fits with a simple perturbative-like expression (4.4) and with the ILM expression (4.5). The results are again not satisfactory. In particular, the ILM expression seems to be strongly disfavoured at $d=0$, while at $d=2$ it looks better than the SVM and perturbative-like expressions. (This suggests that while at $d=0$ the perturbative effects are dominant, at $d=2$ nonperturbative 
effects are surely relevant and cannot be neglected.) By combining the two previous expressions into the ILMp expression (4.6), largely improved best-fits have been obtained. It would be interesting to also have a quantitative numerical prediction from the ILM and see how it compares with the lattice data.

- Finally, we have tried a best-fit with the AdS/CFT expression (4.7), although there is no reason for this formula to apply in our case, since it was originally derived for the $\mathcal{N}=4 \mathrm{SYM}$ theory at strong coupling and large impact parameter. Taking into account that this is a three-parameter best-fit, even this one is not satisfactory, especially at $d=0$. Best-fits with QCD-inspired expressions with only two parameters, like, e.g., the ILMp expression (4.6) [or some appropriate modification of the SVM expression (4.3)] give smaller $\chi_{\text {d.o.f. }}^{2}$.

Although the AdS/CFT expression (4.7), as said above, is not expected to describe real QCD, it nevertheless shows how a non-trivial high-energy behaviour could emerge from a simple analytic dependence on the angle $\theta$. However, in this case, after the analytic continuation into Minkowski space-time, it is not possible to pass to the high-energy limit under the integral sign, as the integrand is an oscillating function of the energy, and one should carry over the remaining integrals first. As we have already pointed out, the integration over the distance between the loops should be the relevant one and, depending on the detailed form of the various coefficient functions, a variety of behaviours could emerge. It seems then worth investigating further the dependence of the correlation functions on the relative distance between the loops, as well as on the dependence on the relative angle, as they could combine in a non-trivial way to determine the highenergy behaviour of meson-meson total cross sections. Moreover, as already recalled in the previous Section [see Eq. (4.2)], the study of the transverse-distance dependence of the Euclidean correlation function $\mathcal{G}_{E}$ at $\theta=0, \pi$ would allow to determine the static dipole-dipole potential. These and other related issues will be addressed in future works.

In conclusion, the fact that the existing models are not able to fully explain the lattice data, which inside the errors represent the true QCD expectation, is a motivation to further investigate the problem of soft high-energy scattering, both on the numerical and on the analytical side. We hope that the interplay of numerical simulations in LGT and analytic nonperturbative calculations can lead to a deeper understanding of the longstanding problem of high-energy scattering in strong interactions. 


\section{References}

[1] S. Donnachie, G. Dosch, P. Landshoff and O. Nachtmann, Pomeron Physics and QCD (Cambridge University Press, Cambridge, 2002).

[2] V.S. Fadin, E.A. Kuraev and L.N. Lipatov, Phys. Lett. B 60 (1975) 50;

L.N. Lipatov, Sov. J. Nucl. Phys. 23 (1976) 338;

E.A. Kuraev, L.N. Lipatov and V.S. Fadin, Sov. Phys. JETP 45 (1977) 199;

I.I. Balitsky and L.N. Lipatov, Sov. J. Nucl. Phys. 28 (1978) 822.

[3] M. Froissart, Phys. Rev. 123 (1961) 1053;

A. Martin, Il Nuovo Cimento 42A (1966) 930;

L. Lukaszuk and A. Martin, Il Nuovo Cimento 52A (1967) 122.

[4] W. Heisenberg, Zeitschrift für Physik 133 (1952) 65.

[5] A. Babansky and I. Balitsky, Phys. Rev. D 67 (2003) 054026.

[6] I. Balitsky, in At the frontier of Particle Physics - Handbook of QCD (Boris Ioffe Festschrift), edited by M. Shifman (World Scientific, Singapore, 2001), vol. 2, 12371342; hep-ph/0101042.

[7] A.B. Kaidalov, in At the frontier of Particle Physics - Handbook of QCD (Boris Ioffe Festschrift), edited by M. Shifman (World Scientific, Singapore, 2001), vol. 1, 603-636; hep-ph/0103011.

[8] O. Nachtmann, Ann. Phys. 209 (1991) 436.

[9] H.G. Dosch, E. Ferreira and A. Krämer, Phys. Rev. D 50 (1994) 1992.

[10] O. Nachtmann, in Perturbative and Nonperturbative aspects of Quantum Field Theory, edited by H. Latal and W. Schweiger (Springer-Verlag, Berlin, Heidelberg, 1997).

[11] E.R. Berger and O. Nachtmann, Eur. Phys. J. C 7 (1999) 459.

[12] H.G. Dosch, in At the frontier of Particle Physics - Handbook of QCD (Boris Ioffe Festschrift), edited by M. Shifman (World Scientific, Singapore, 2001), vol. 2, 11951236.

[13] A.I. Shoshi, F.D. Steffen and H.J. Pirner, Nucl. Phys. A 709 (2002) 131. 
[14] E. Meggiolaro, Z. Phys. C 76 (1997) 523.

[15] E. Meggiolaro, Eur. Phys. J. C 4 (1998) 101.

[16] E. Meggiolaro, Nucl. Phys. B 625 (2002) 312.

[17] E. Meggiolaro, Nucl. Phys. B 707 (2005) 199.

[18] A.I. Shoshi, F.D. Steffen, H.G. Dosch and H.J. Pirner, Phys. Rev. D 68 (2003) 074004 .

[19] E. Shuryak and I. Zahed, Phys. Rev. D 62 (2000) 085014.

[20] R.A. Janik and R. Peschanski, Nucl. Phys. B 565 (2000) 193.

[21] R.A. Janik and R. Peschanski, Nucl. Phys. B 586 (2000) 163.

[22] R.A. Janik, Phys. Lett. B 500 (2001) 118.

[23] E. Meggiolaro, Phys. Lett. B 651 (2007) 177.

[24] H. Verlinde and E. Verlinde, hep-th/9302104.

[25] I.I. Balitsky and L.N. Lipatov, Sov. J. Nucl. Phys. 28 (1978) 822;

I.I. Balitsky and L.N. Lipatov, JETP Letters 30 (1979) 355.

[26] M. Giordano and E. Meggiolaro, Phys. Rev. D 74 (2006) 016003.

[27] J.E. Bresenham, IBM Sys. Jour. 4 (1965) 25.

[28] B. Bolder, T. Struckmann, G.S. Bali, N. Eicker, T. Lippert, B. Orth, K. Schilling, P. Ueberholz, Phys. Rev. D 63 (2001) 074504.

[29] M. Creutz, Phys. Rev. D 21 (1980) 2308.

[30] N. Cabibbo, E. Marinari, Phys. Lett. B 119 (1982) 387.

[31] A.D. Kennedy, B.J. Pendleton, Phys. Lett. B 156 (1985) 393.

[32] M. Creutz, Phys. Rev. D 36 (1987) 515.

[33] K.G. Wilson, Phys. Rev. D 10 (1974) 2445. 
[34] C. Michael, M. Teper, Phys. Lett. B 206 (1988) 299;

G.S. Bali, K. Schilling, Phys. Rev. D 47 (1993) 661;

M. Guagnelli, R. Sommer and H. Wittig, Nucl. Phys. B 535 (1998) 389.

[35] T. Appelquist and W. Fischler, Phys. Lett. B 77 (1978) 405;

G. Bhanot, W. Fischler and S. Rudaz, Nucl. Phys. B 155 (1979) 208;

M.E. Peskin, Nucl. Phys. B 156 (1979) 365;

G. Bhanot and M.E. Peskin, Nucl. Phys. B 156 (1979) 391.

[36] H.G. Dosch, M. Rueter, Phys. Lett. B 380 (1996) 177. 


\section{FIGURE CAPTIONS}

Fig. 1 The space-time configuration of the two Wilson loops $\mathcal{W}_{1}$ and $\mathcal{W}_{2}$ entering in the expression for the dipole-dipole elastic scattering amplitude in the high-energy limit.

Fig. 2 The analyticity domains of the function $\overline{\mathcal{G}}_{E}$ in the complex variable $\theta$ and of the function $\overline{\mathcal{G}}_{M}$ in the complex variable $\chi$.

Fig. 3 The minimal-distance prescription for a line with slope $\tan \theta=1 / 2$.

Fig. 4 The relevant Wilson-loop configuration. Using the $O(4)$ invariance of the Euclidean theory we have put $p_{1 E}$ parallel to the $x_{E 1}$ axis.

Fig. 5 Loop configuration in the transverse plane. In the "ave" case the link orientation is not shown as it is averaged over.

Fig. 6 Dependence of $\mathcal{G}_{E}$ on the length $L_{1}=L_{2}=L$ (in lattice units) of the loops at $\theta=90^{\circ}$ for $d=0,1,2$.

Fig. 7 Angular dependence of $\mathcal{G}_{E}$ for various lengths of the loops, for $d=0$. The numbers in the key refer to the on-axis loop length and to the off-axis loop set respectively, as explained in the text and in Table 1. (Different data sets have been slightly shifted horizontally for clarity.)

Fig. 8 Angular dependence of $\mathcal{G}_{E}$ for various lengths of the loops, for $d=1$. The notation is the same as in Fig. 7 .

Fig. 9 Angular dependence of $\mathcal{G}_{E}$ for various lengths of the loops, for $d=2$. The notation is the same as in Fig. 7 .

Fig. 10 Dependence of $\mathcal{C}_{E}(d)$ (on a logarithmic scale) on the distance $d$ (in lattice units) at $\theta=45^{\circ}$ and $\theta=90^{\circ}$.

Fig. 11 Comparison of the lattice data to the SVM prediction (4.3) with $K_{\text {SvM }}$ calculated according to Ref. [18] (solid line) and to the one-parameter $\left(K_{\mathrm{SVM}}\right)$ best-fit (for the "zzz" and "zyy" cases only) with the SVM expression (4.3) (dotted line) at $d=0$.

Fig. 12 The same comparison as in Fig. 11, but at $d=1$. 
Fig. 13 The same comparison as in Fig. 11, but at $d=2$.

Fig. 14 Comparison of lattice data to best-fits with the perturbative-like expression (4.4) (solid line), the ILM expression (4.5) (dotted line) and the ILMp expression (4.6) (dashed line) at $d=0$.

Fig. 15 The same comparison as in Fig. 14, but at $d=1$.

Fig. 16 Comparison of lattice data to a best-fit with the AdS/CFT expression (4.7) for various cases.

Fig. 17 The crossing-antisymmetric component $\mathcal{C}_{E}^{-}$, as defined in Eq. (4.8), for the "zzz/zyy" case at $d=0$ and the corresponding prediction using the SVM expression (4.3) (solid line). 


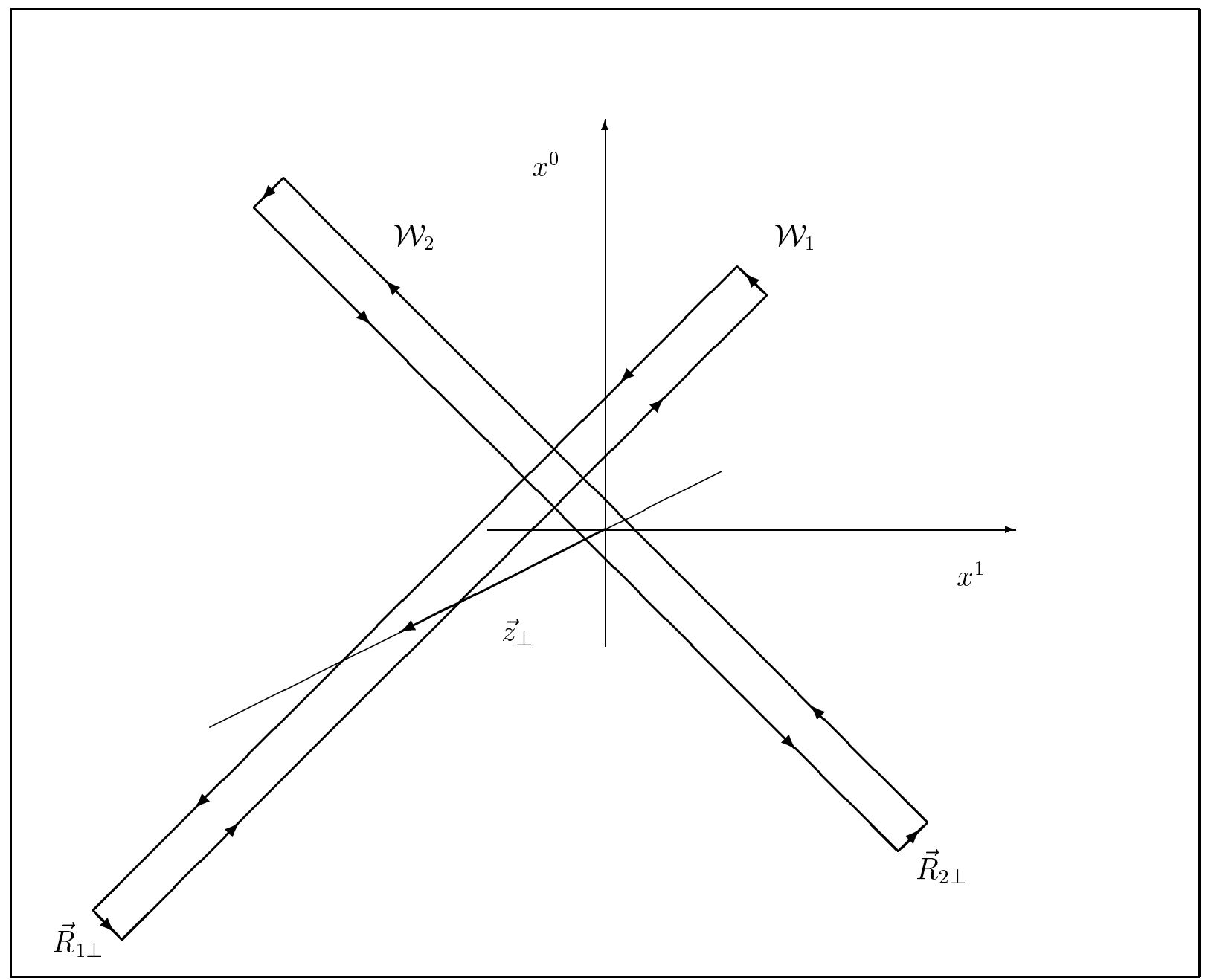

Figure 1: The space-time configuration of the two Wilson loops $\mathcal{W}_{1}$ and $\mathcal{W}_{2}$ entering in the expression for the dipole-dipole elastic scattering amplitude in the high-energy limit. 

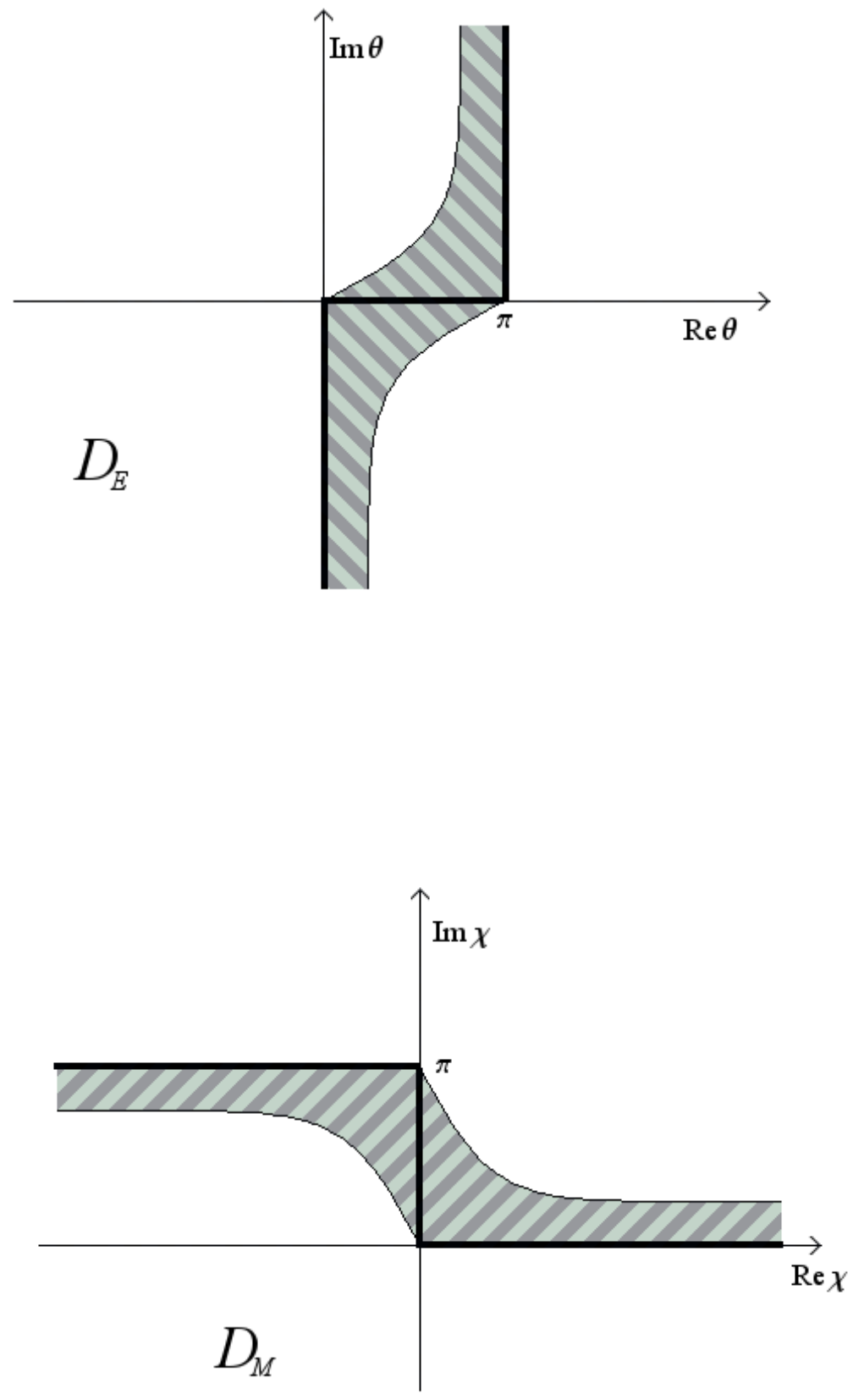

Figure 2: The analyticity domains of the function $\overline{\mathcal{G}}_{E}$ in the complex variable $\theta$ and of the function $\overline{\mathcal{G}}_{M}$ in the complex variable $\chi$. 


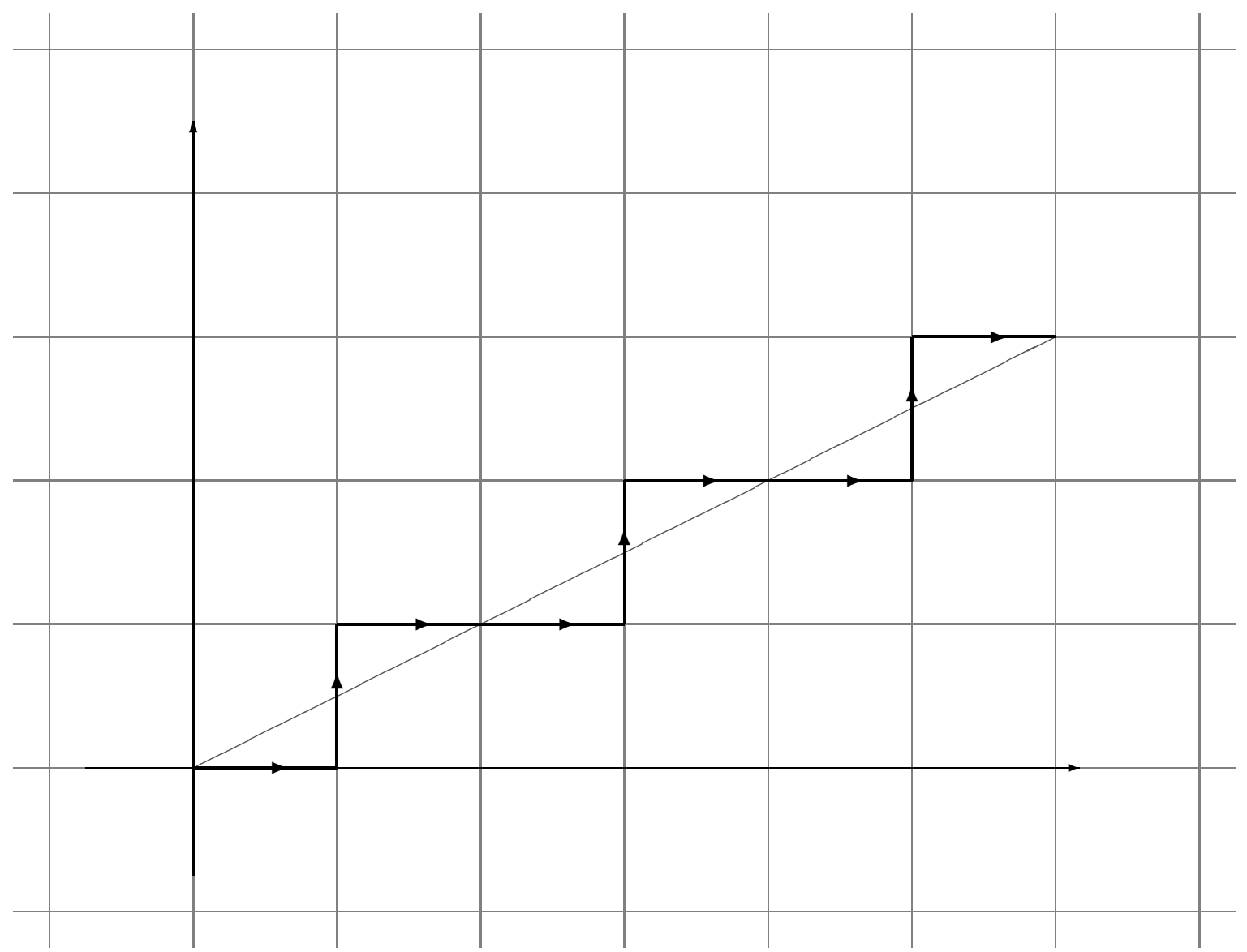

Figure 3: The minimal-distance prescription for a line with slope $\tan \theta=1 / 2$. 


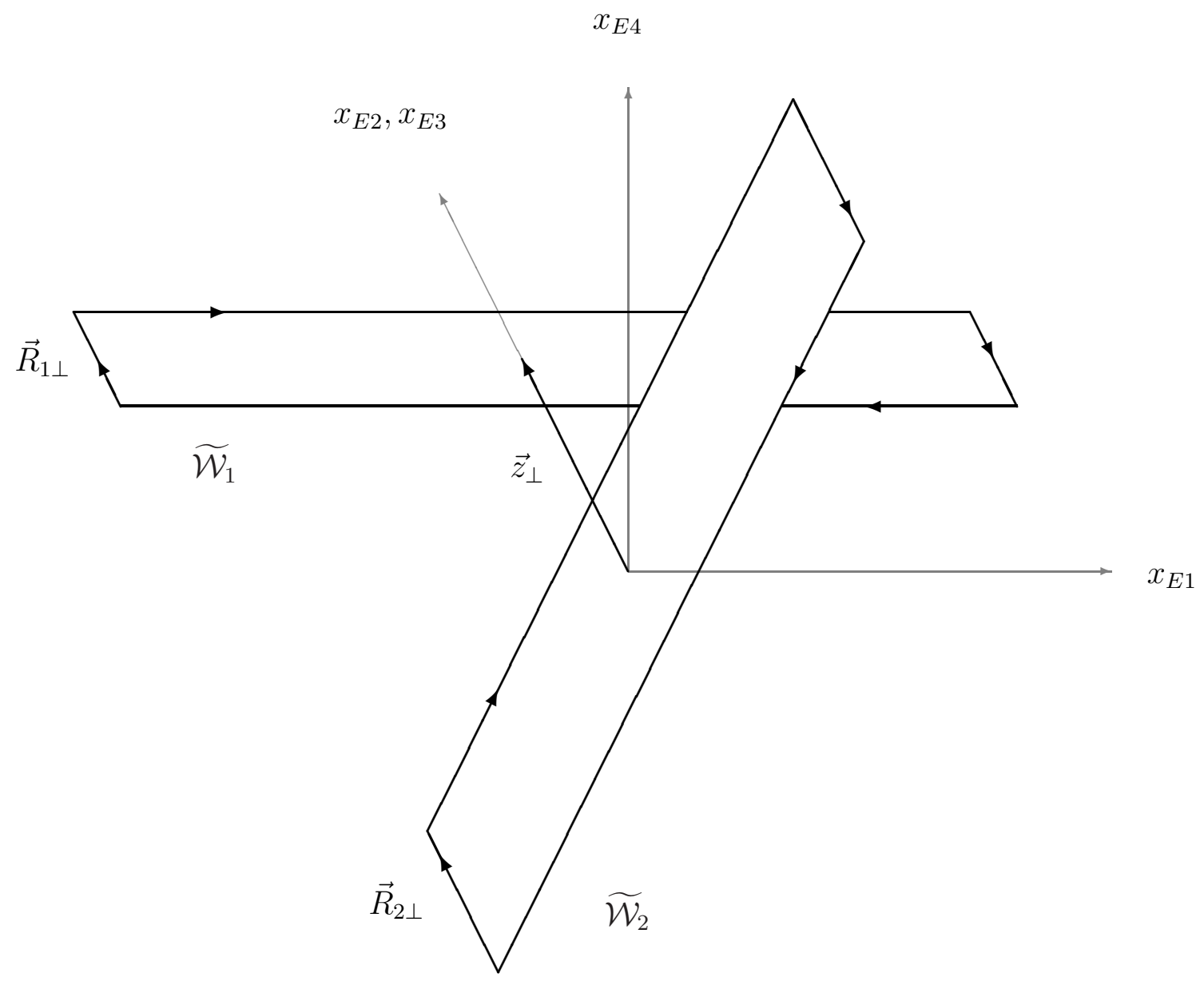

Figure 4: The relevant Wilson-loop configuration. Using the $O(4)$ invariance of the Euclidean theory we have put $p_{1 E}$ parallel to the $x_{E 1}$ axis. 


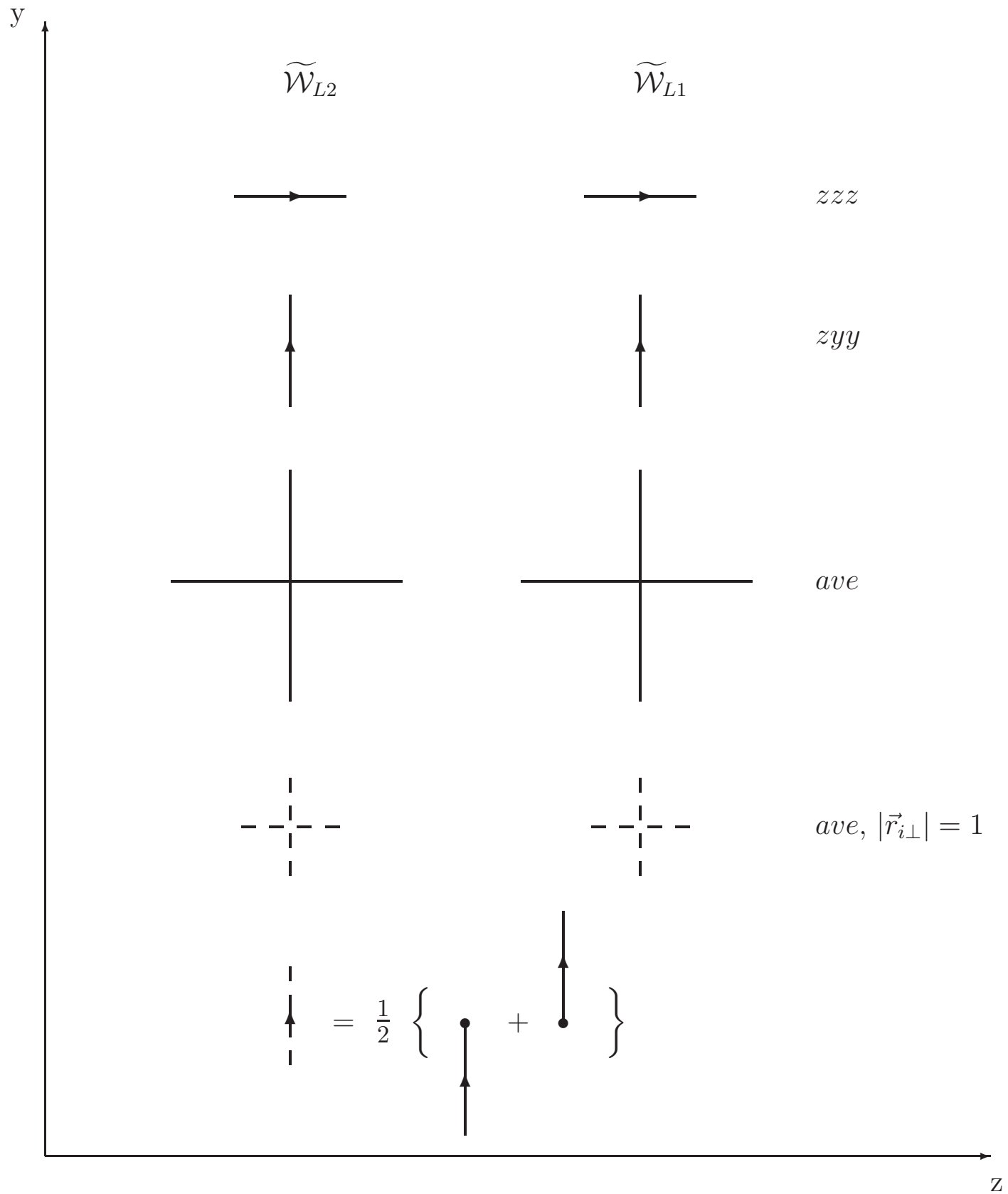

Figure 5: Loop configuration in the transverse plane. In the "ave" case the link orientation is not shown as it is averaged over. 

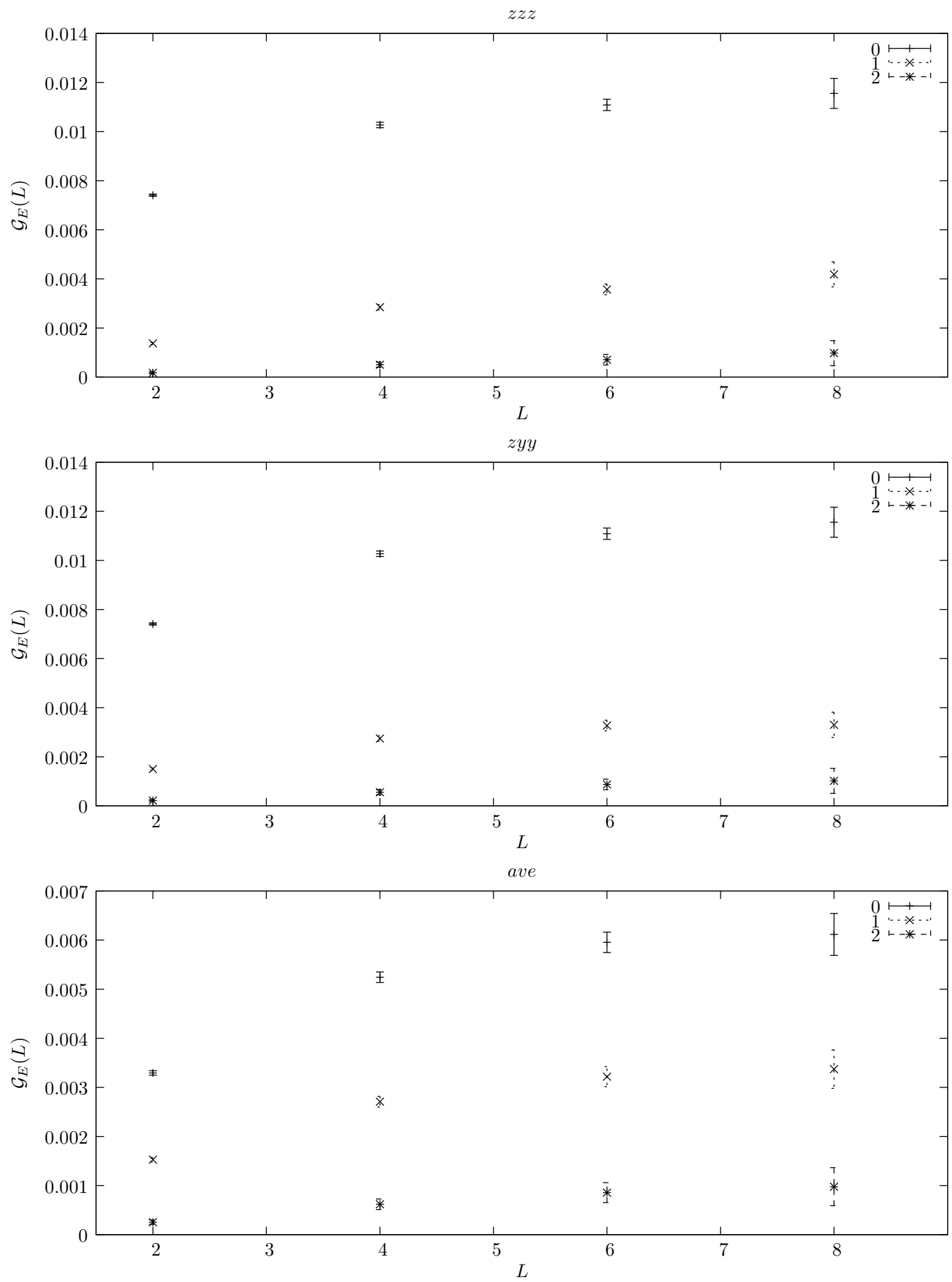

Figure 6: Dependence of $\mathcal{G}_{E}$ on the length $L_{1}=L_{2}=L$ (in lattice units) of the loops at $\theta=90^{\circ}$ for $d=0,1,2$. 

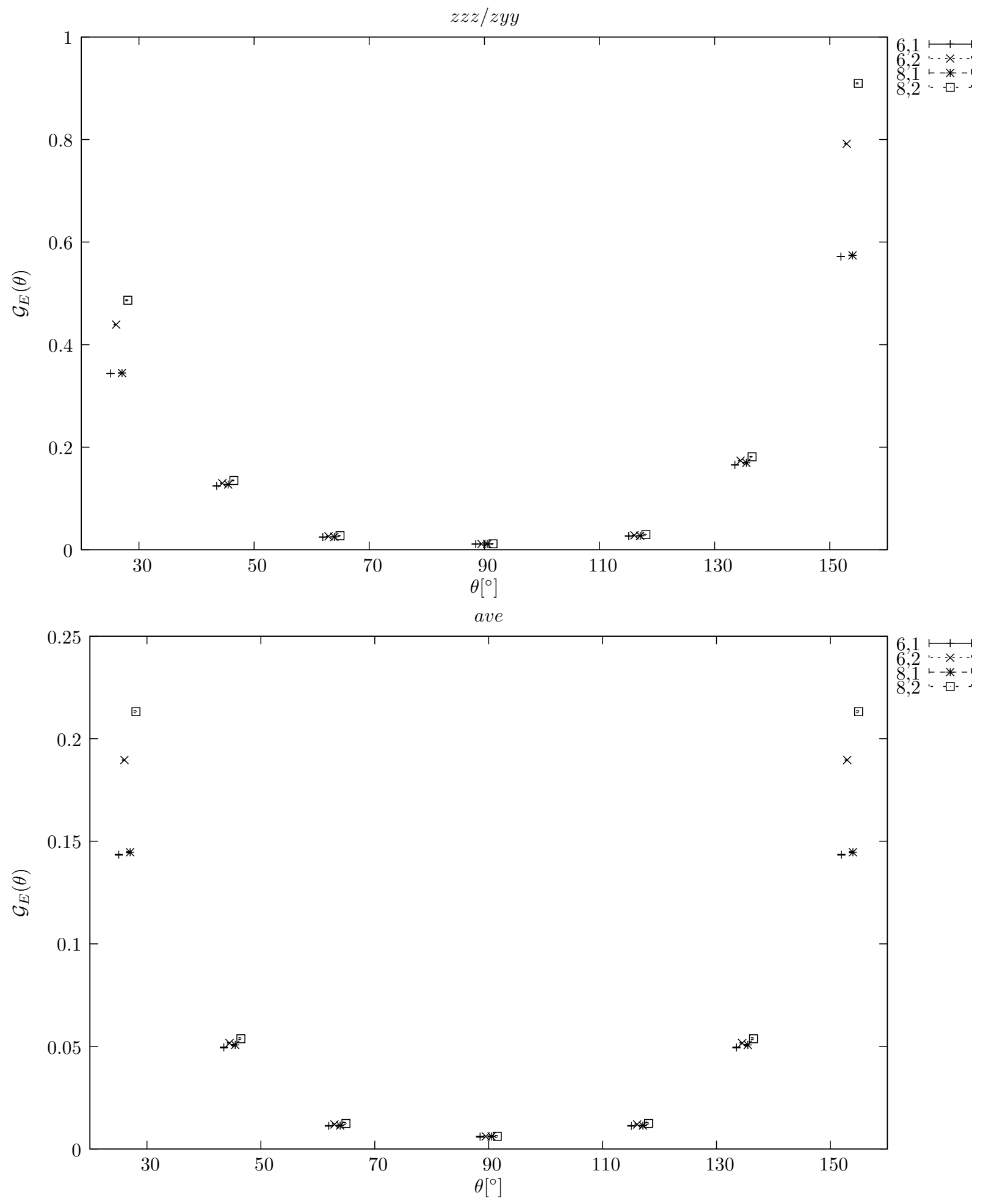

Figure 7: Angular dependence of $\mathcal{G}_{E}$ for various lengths of the loops, for $d=0$. The numbers in the key refer to the on-axis loop length and to the off-axis loop set respectively, as explained in the text and in Table 1. (Different data sets have been slightly shifted horizontally for clarity.) 


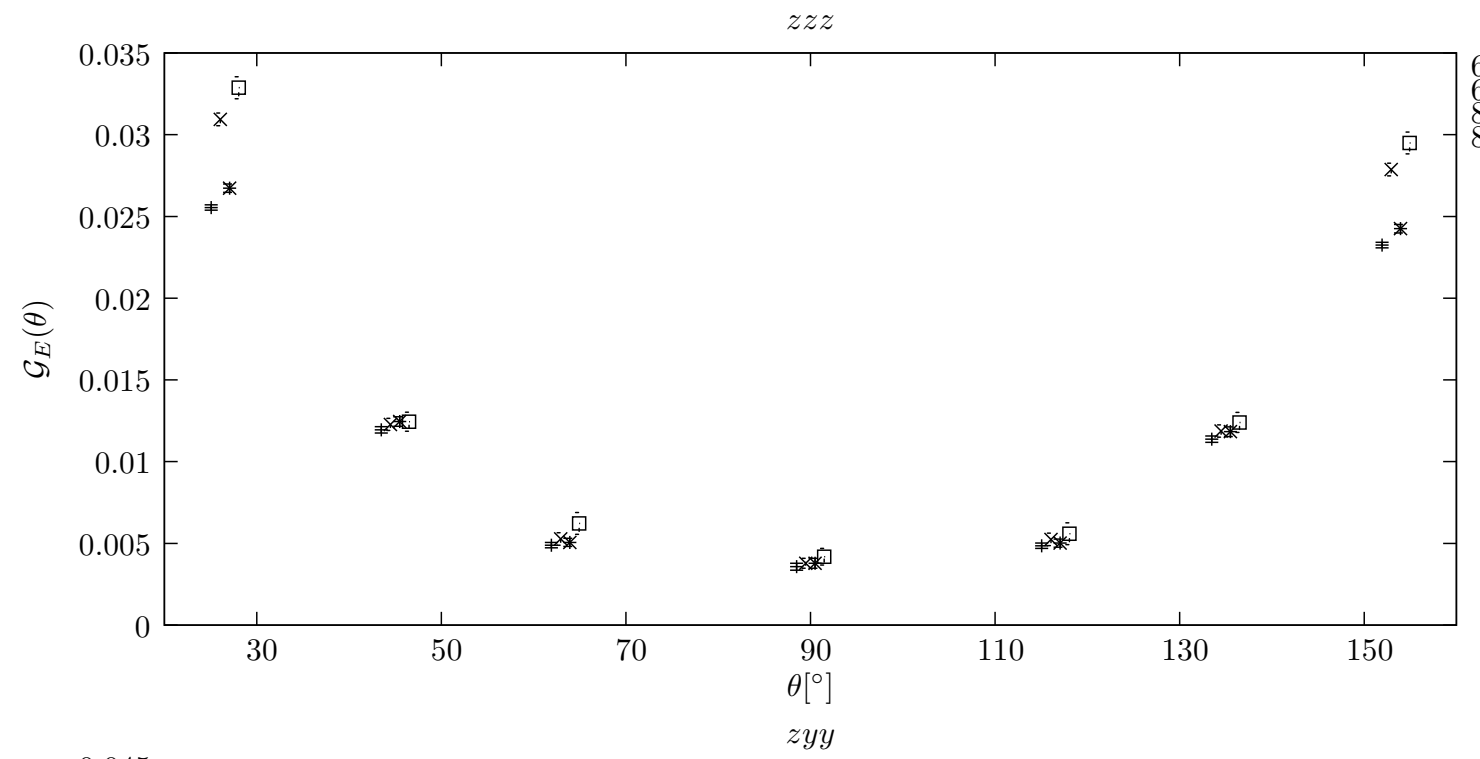

$6,1 \longmapsto$

8,

8,1 เ - * -

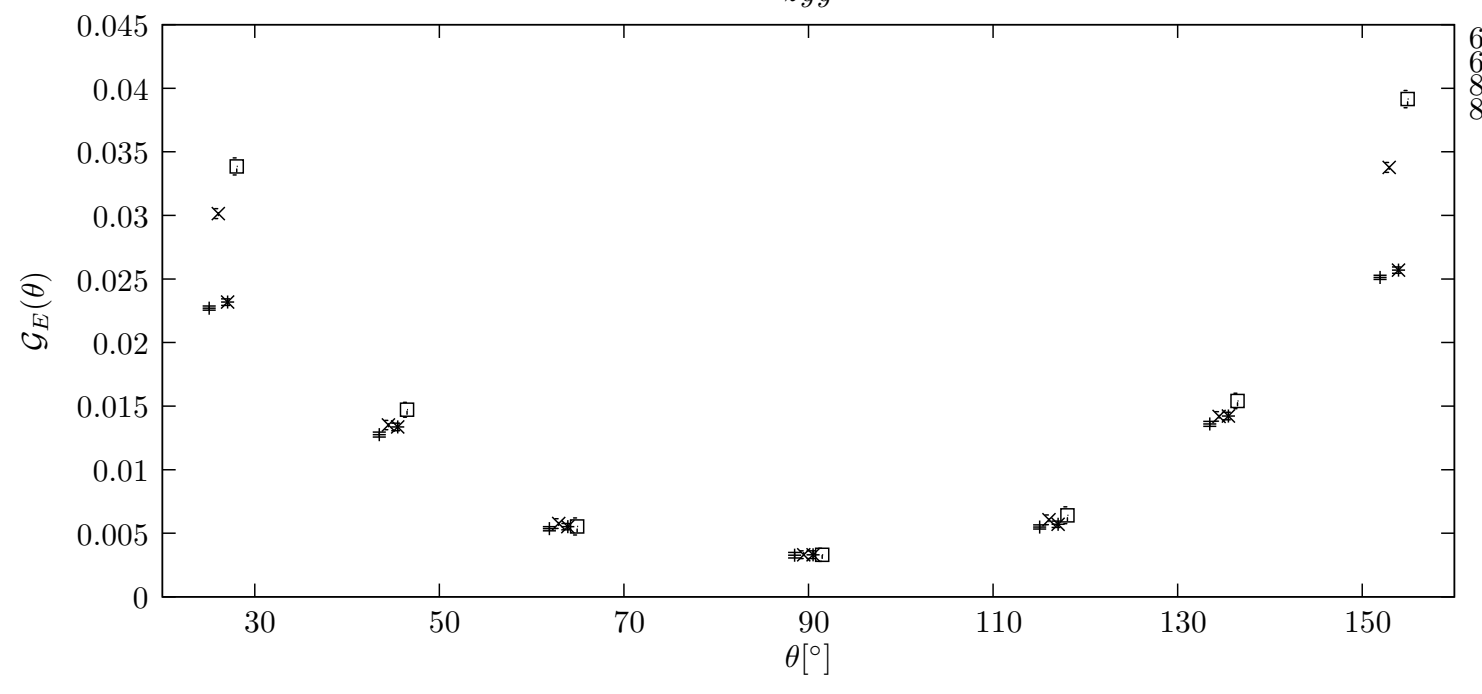

$6,1 \longmapsto$

$8,1 \vdash-*-1$

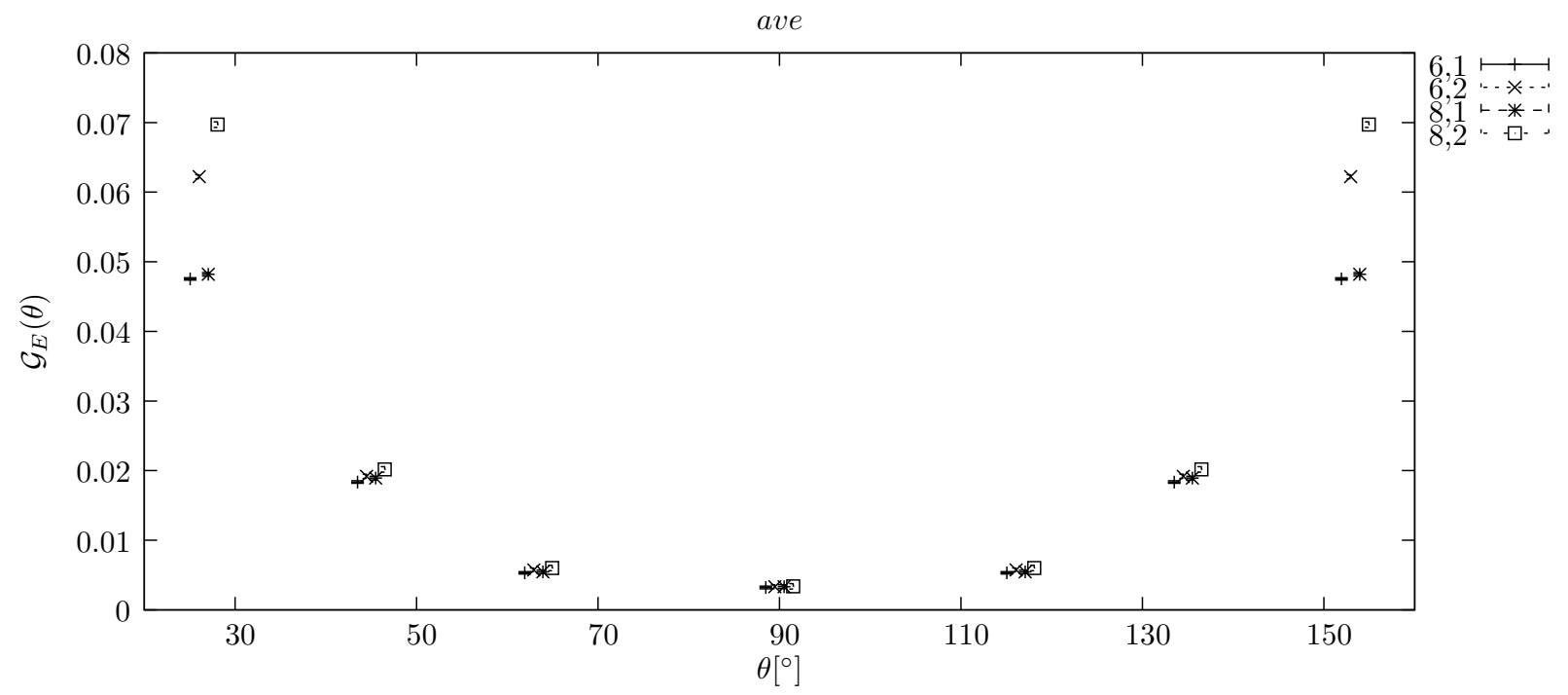

Figure 8: Angular dependence of $\mathcal{G}_{E}$ for various lengths of the loops, for $d=1$. The notation is the same as in Fig. 7 . 


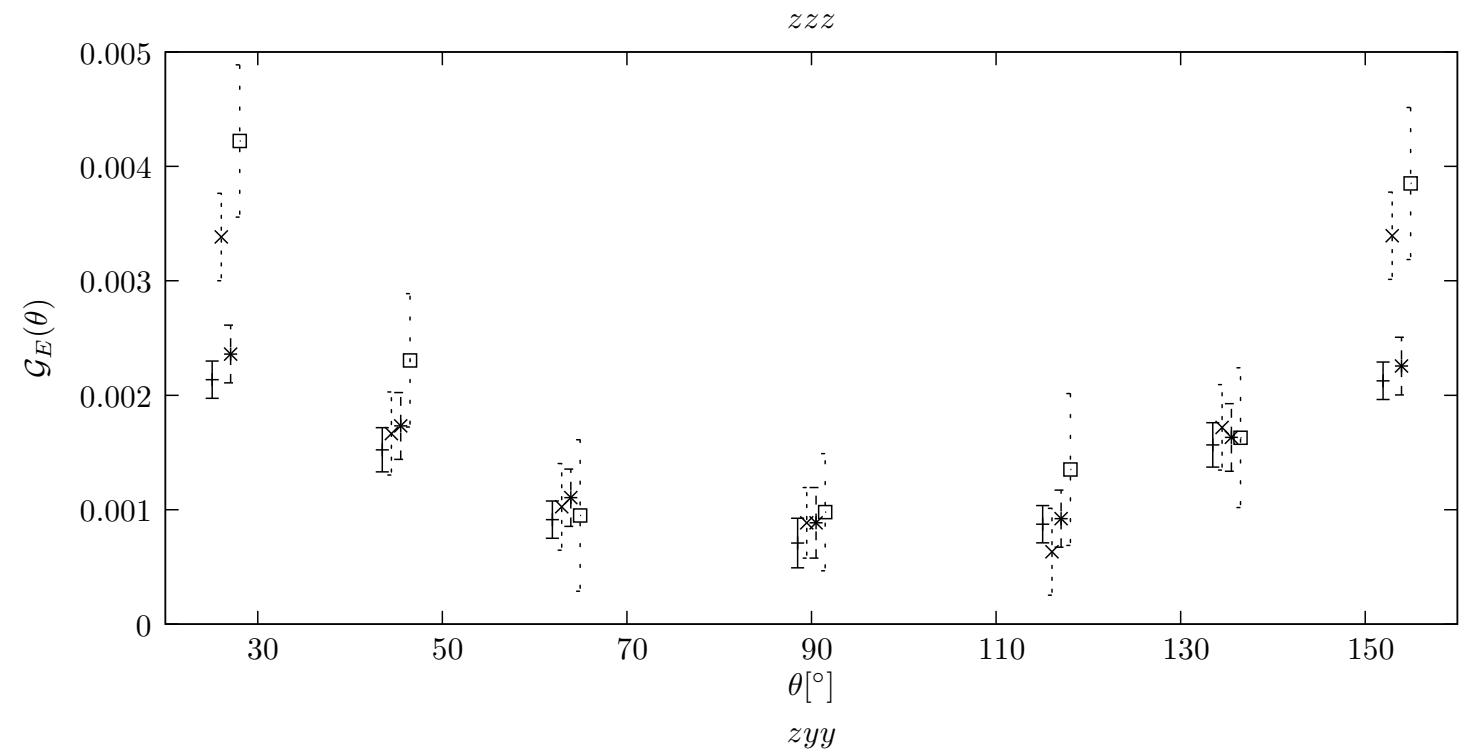

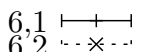

$8,1+-*-1$
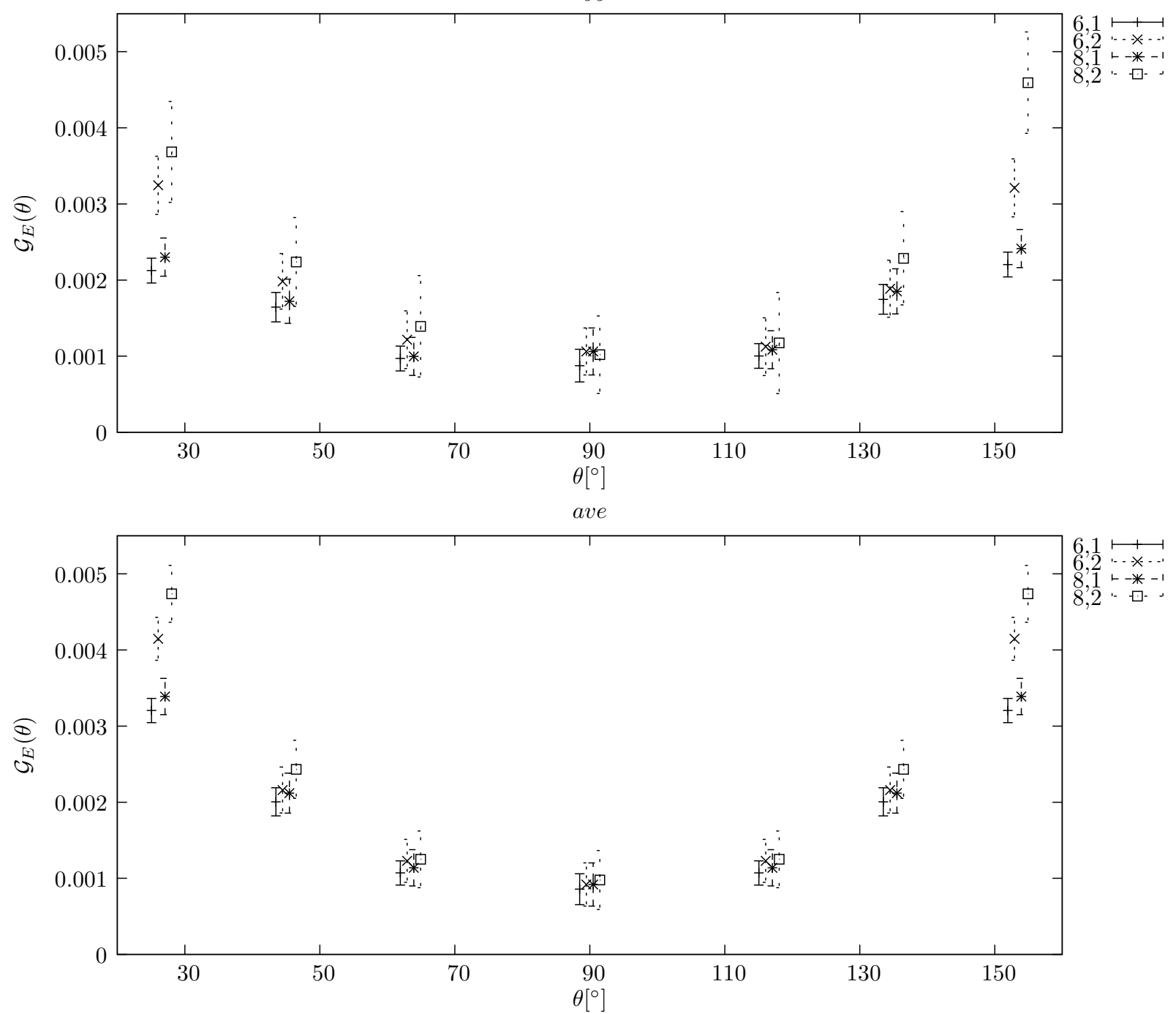

Figure 9: Angular dependence of $\mathcal{G}_{E}$ for various lengths of the loops, for $d=2$. The notation is the same as in Fig. 7. 

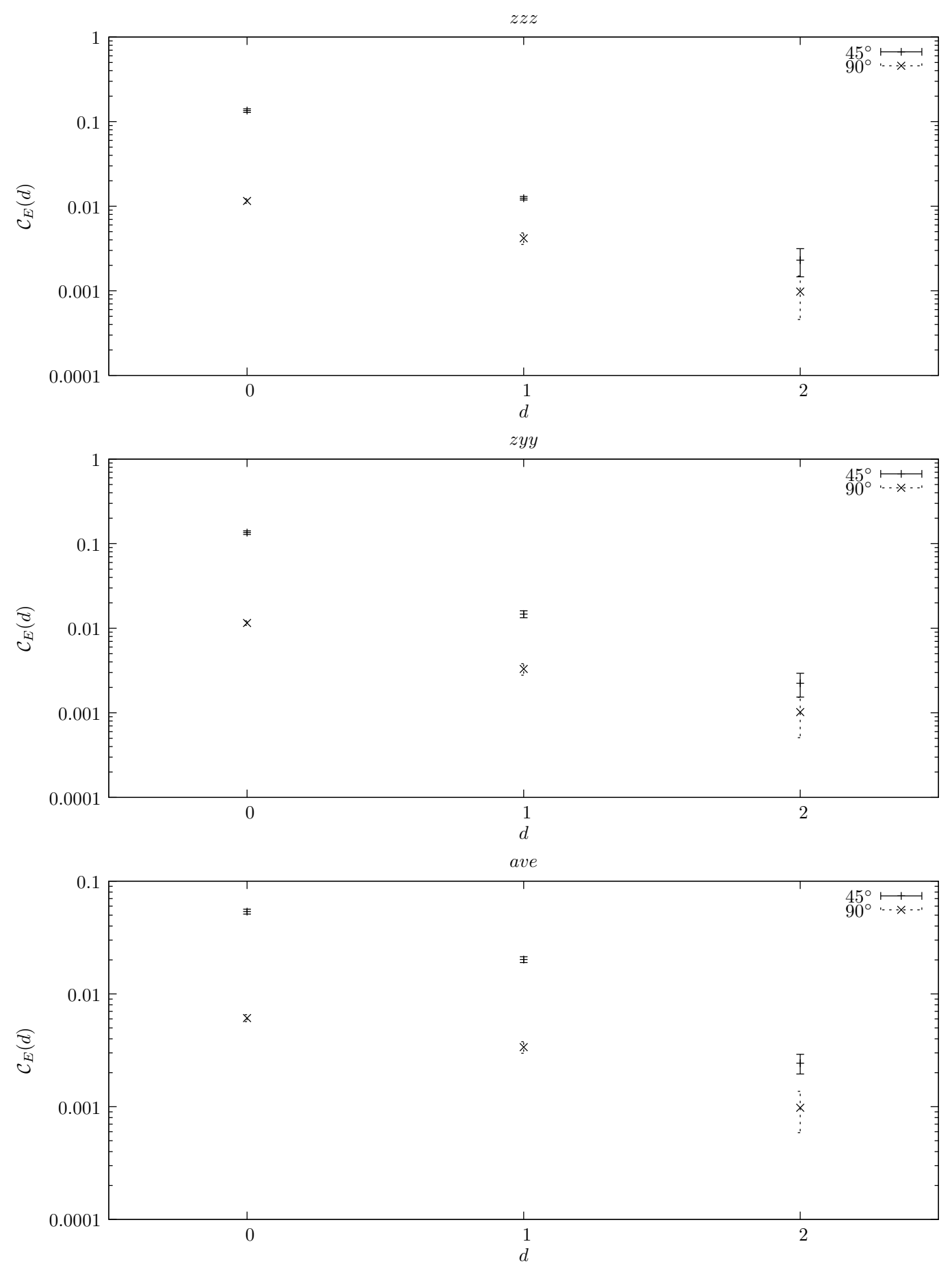

Figure 10: Dependence of $\mathcal{C}_{E}(d)$ (on a logarithmic scale) on the distance $d$ (in lattice units) at $\theta=45^{\circ}$ and $\theta=90^{\circ}$. 

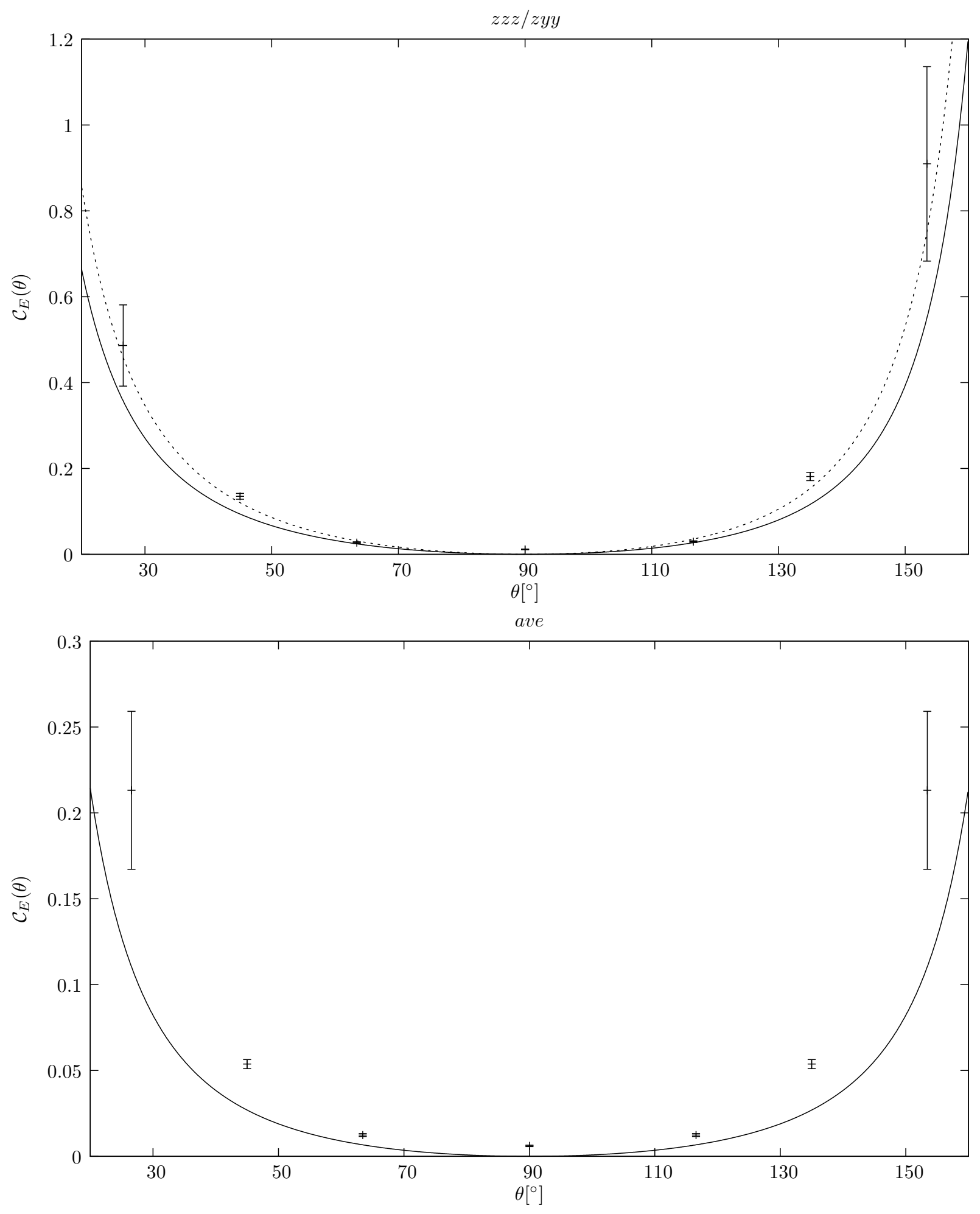

Figure 11: Comparison of the lattice data to the SVM prediction (4.3) with $K_{\mathrm{SVM}}$ calculated according to Ref. [18] (solid line) and to the one-parameter $\left(K_{\mathrm{SVM}}\right)$ best-fit (for the " $z z z$ " and "zyy" cases only) with the SVM expression (4.3) (dotted line) at $d=0$. 

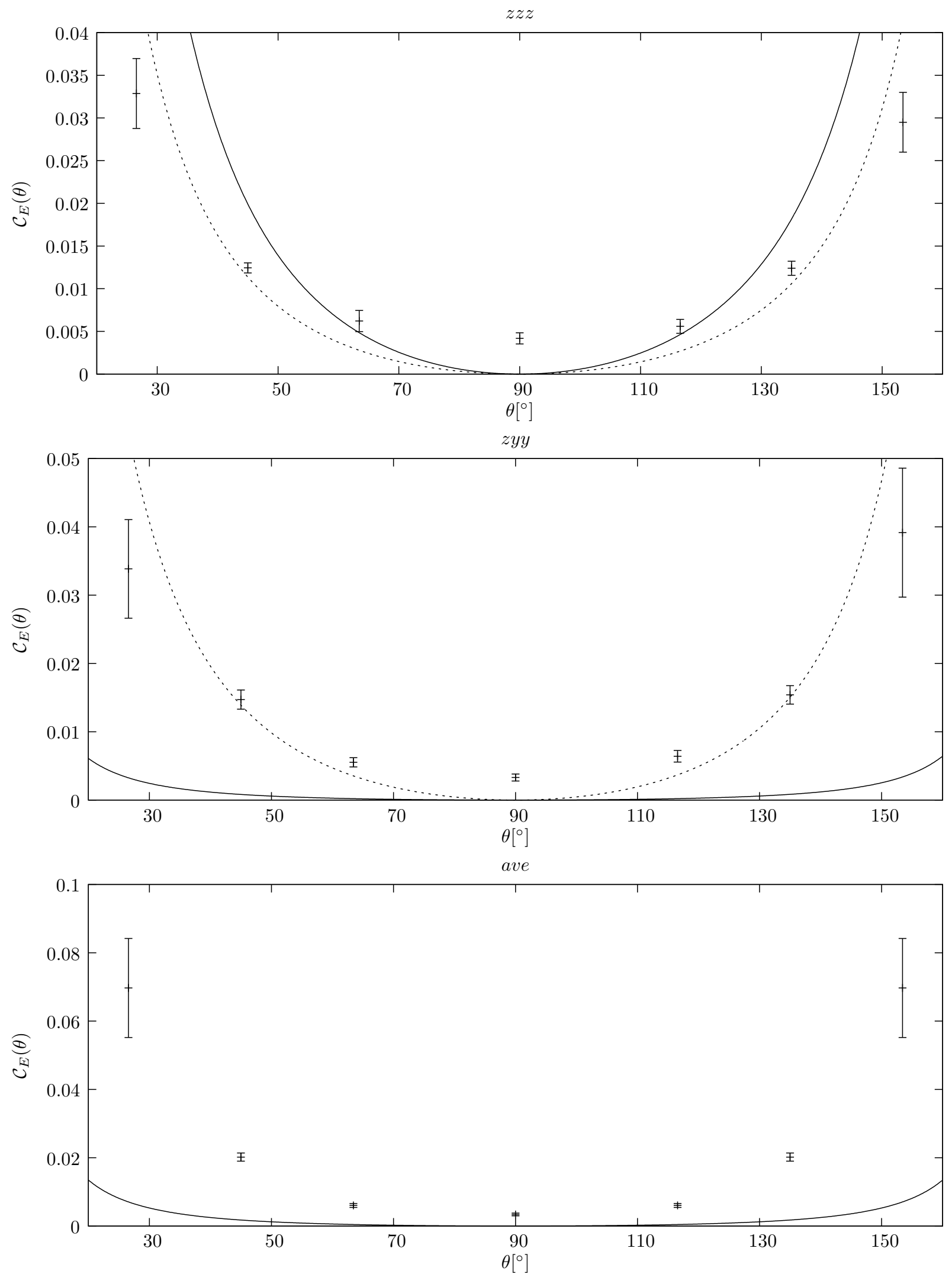

Figure 12: The same comparison as in Fig. 11, but at $d=1$. 

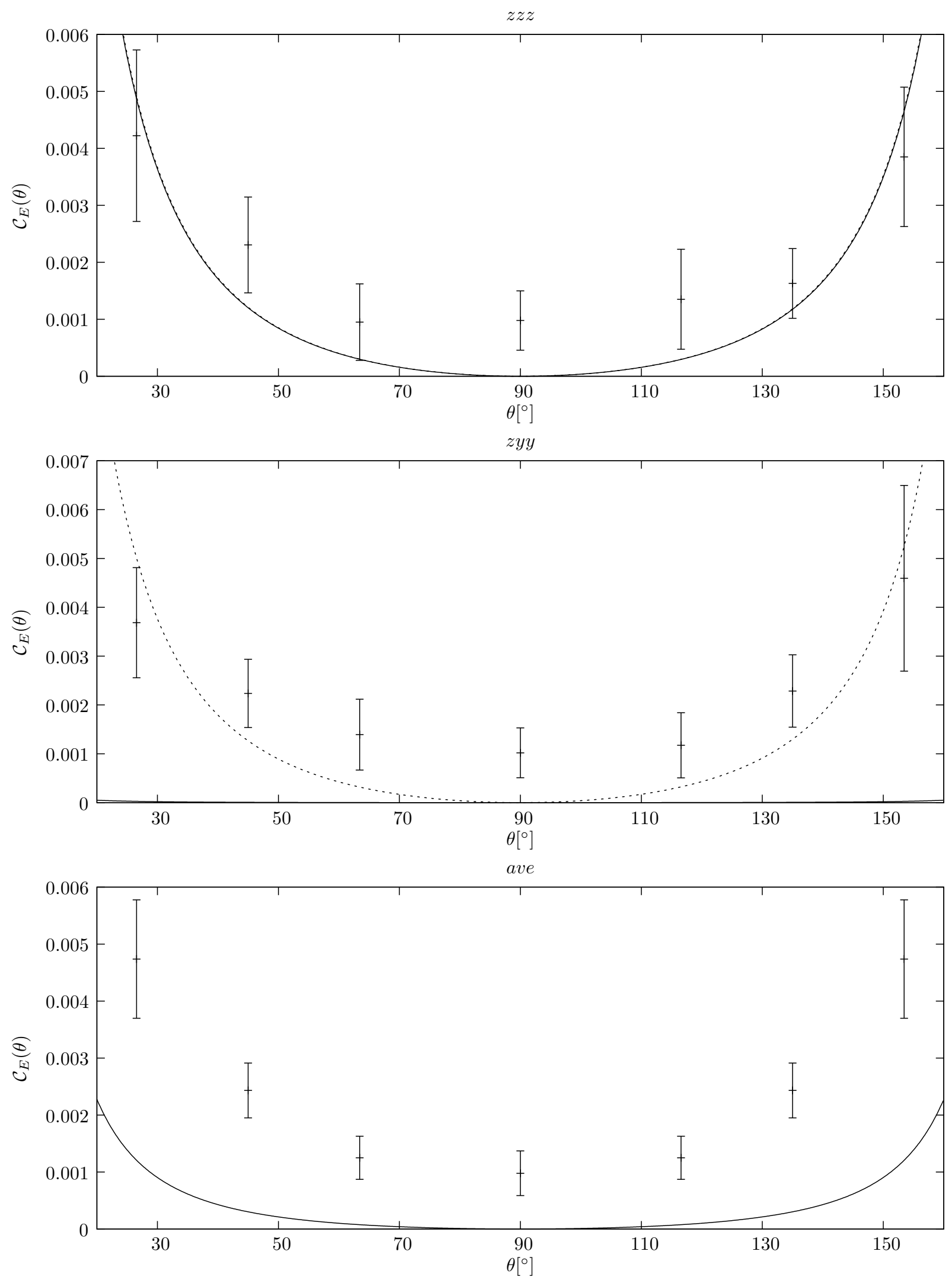

Figure 13: The same comparison as in Fig. 11, but at $d=2$. 

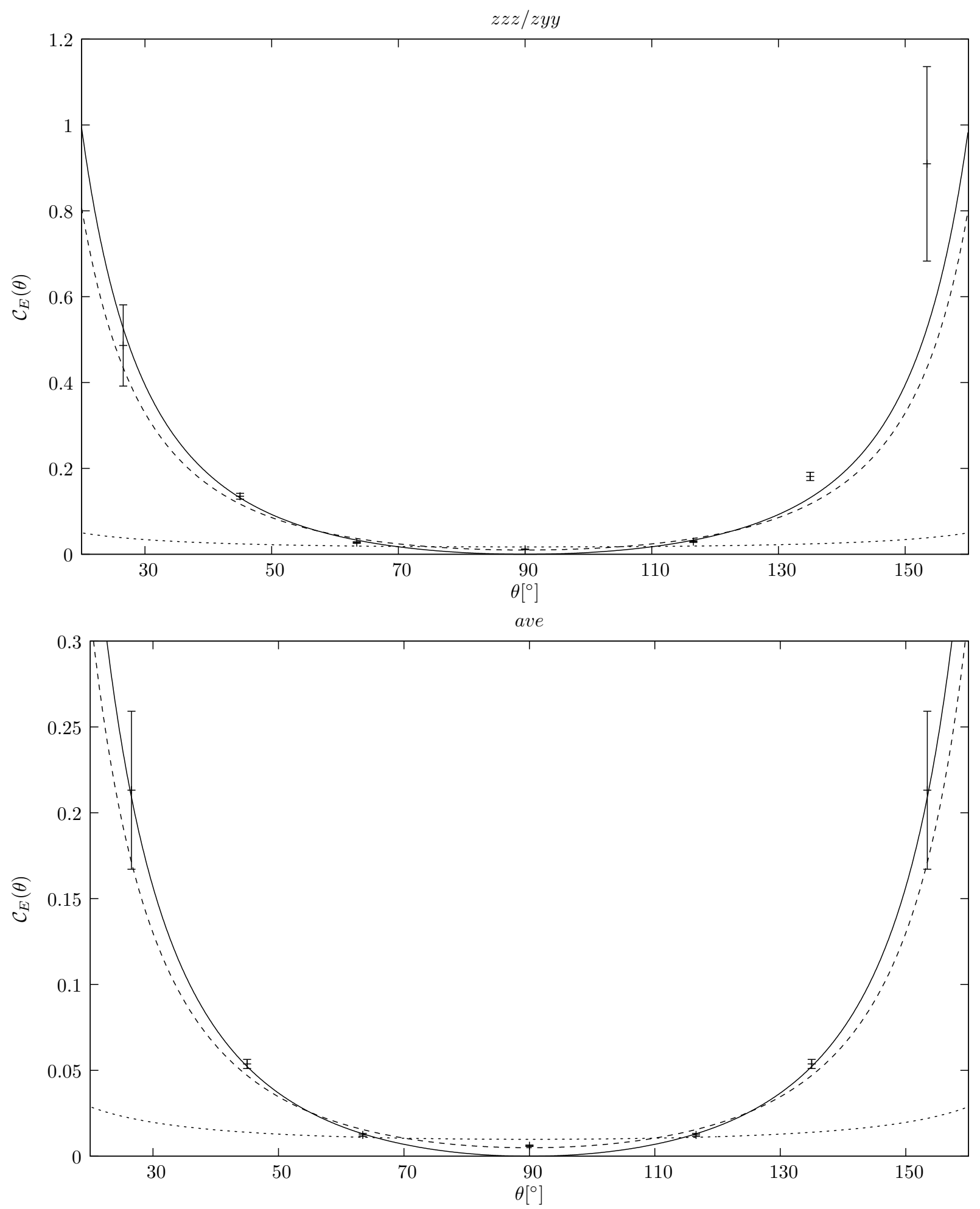

Figure 14: Comparison of lattice data to best-fits with the perturbative-like expression (4.4) (solid line), the ILM expression (4.5) (dotted line) and the ILMp expression (4.6) (dashed line) at $d=0$. 

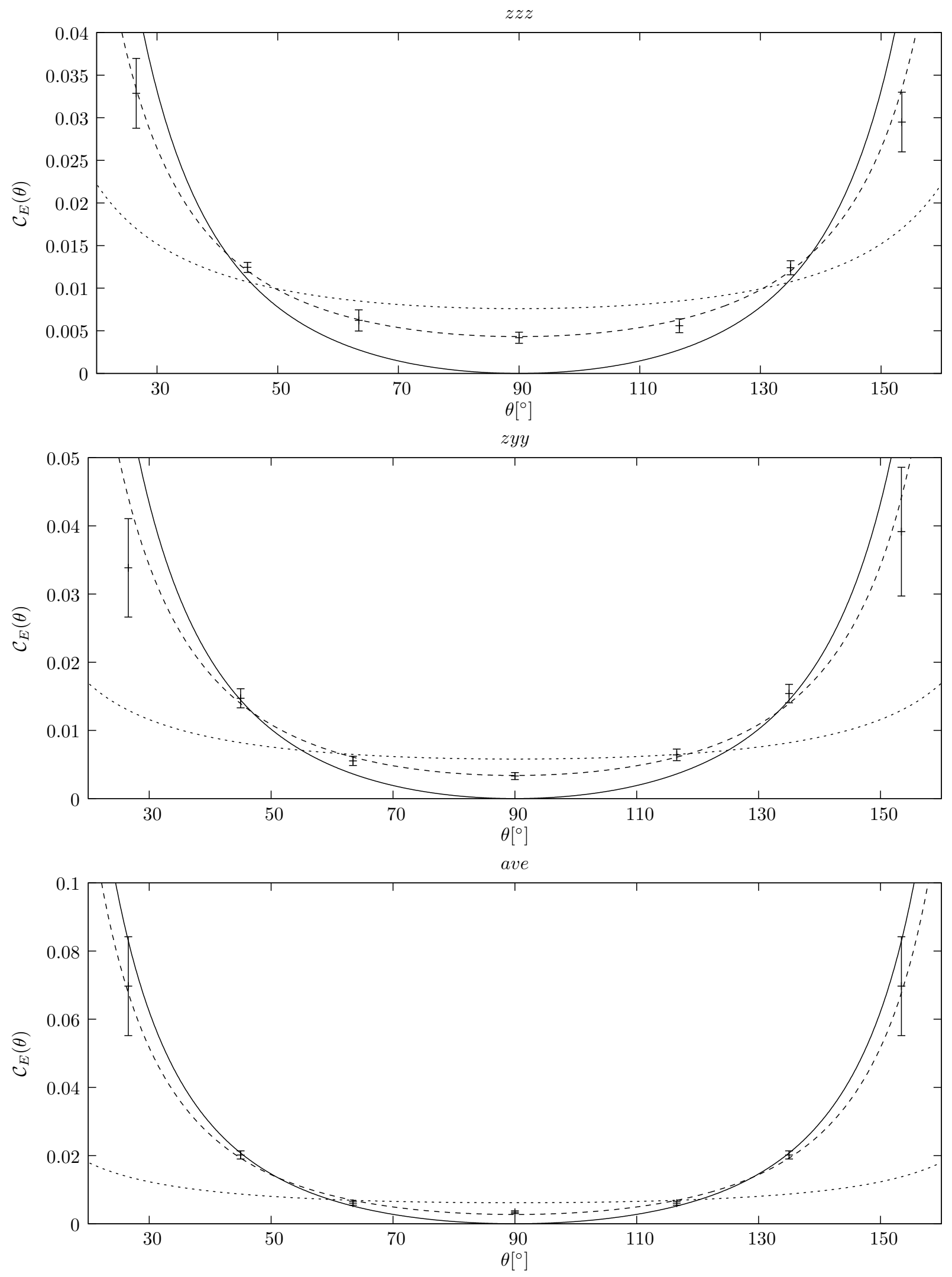

Figure 15: The same comparison as in Fig. 14, but at $d=1$. 

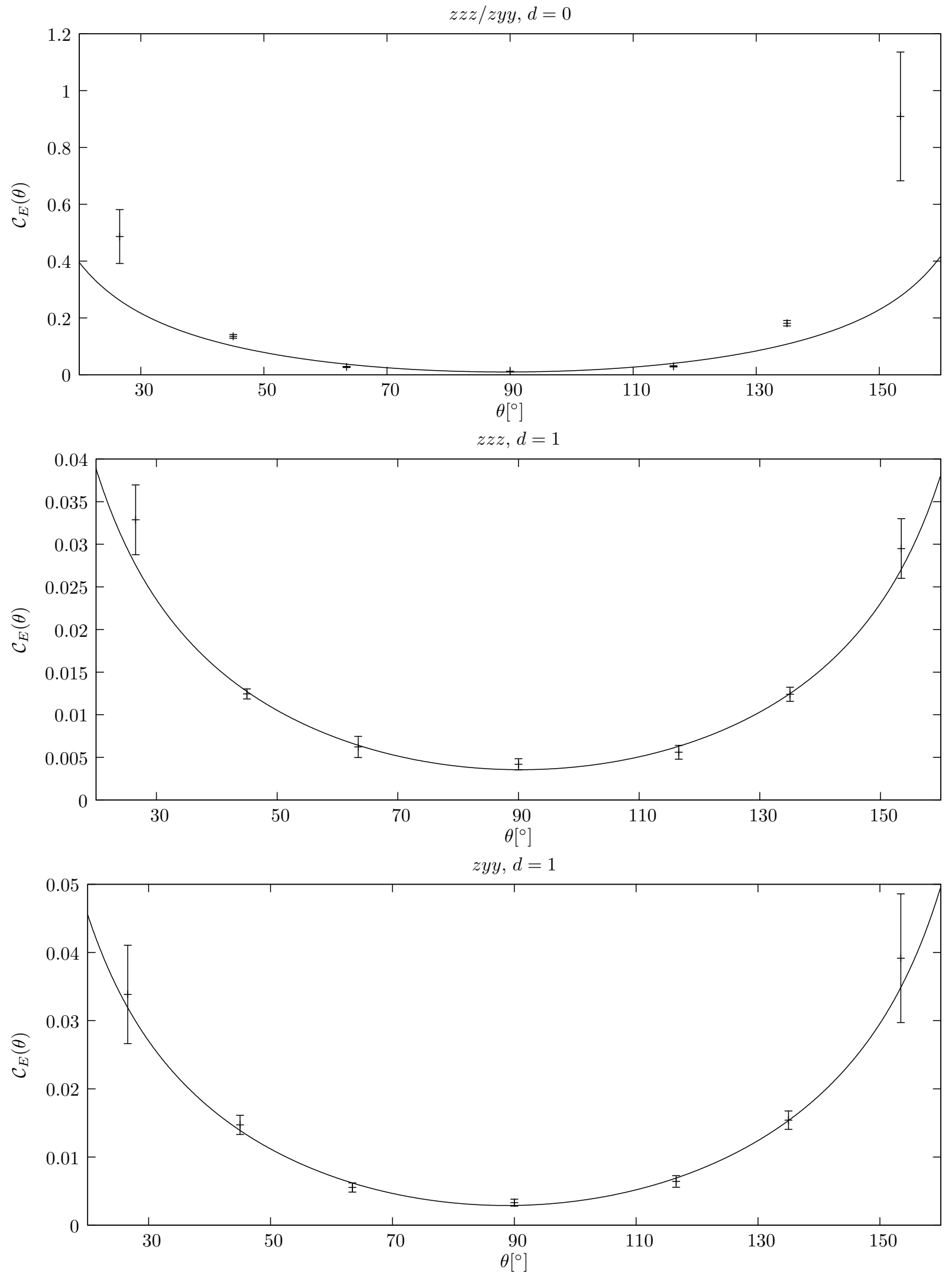

Figure 16: Comparison of lattice data to a best-fit with the AdS/CFT expression (4.7) for various cases. 


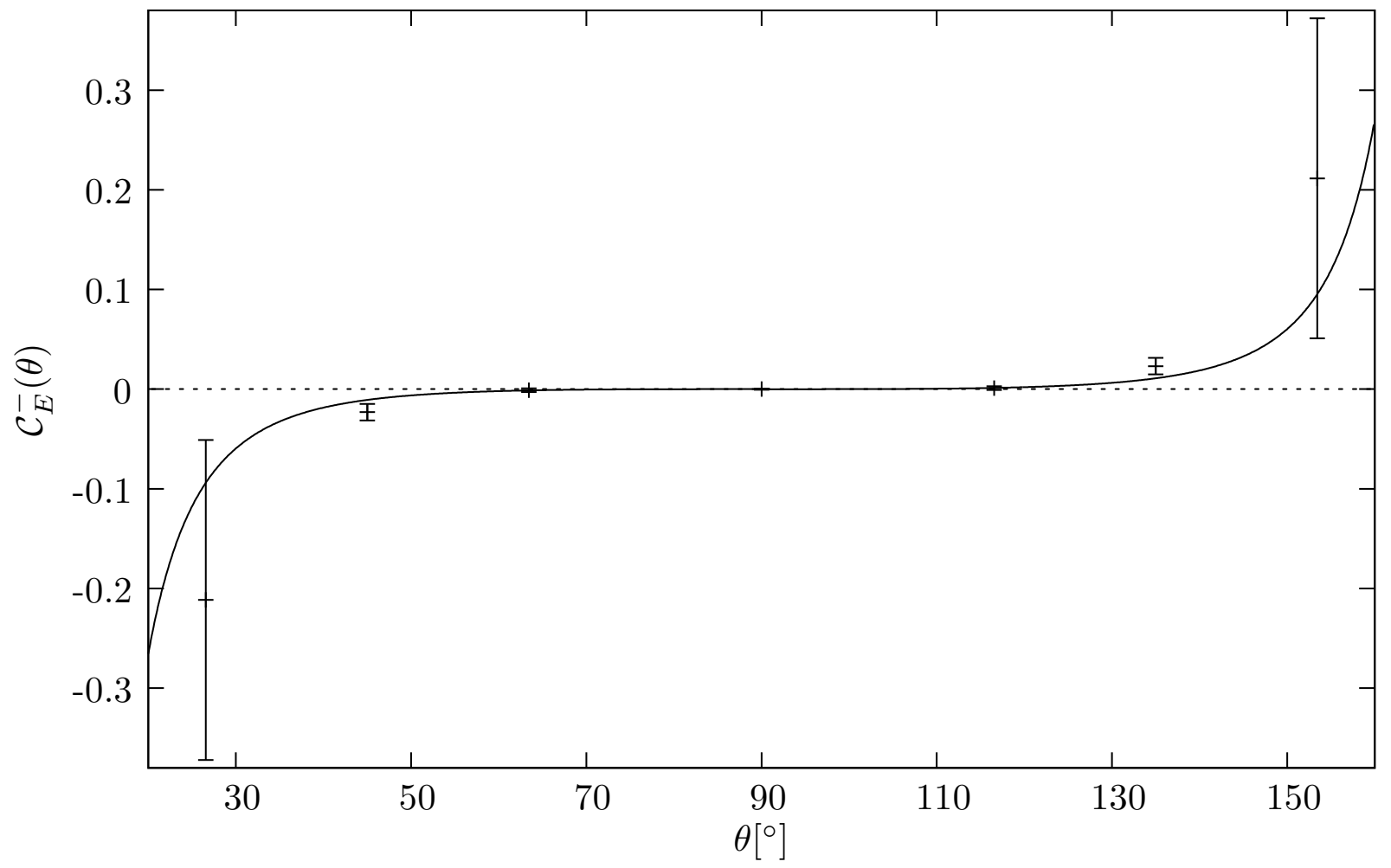

Figure 17: The crossing-antisymmetric component $\mathcal{C}_{E}^{-}$, as defined in Eq. (4.8), for the "zzz/zyy" case at $d=0$ and the corresponding prediction using the SVM expression (4.3) (solid line). 